\title{
LARGE DEVIATION PRINCIPLE FOR BENEDICKS-CARLESON QUADRATIC MAPS
}

\author{
YONG MOO CHUNG AND HIROKI TAKAHASI
}

\begin{abstract}
Since the pioneering works of Jakobson and Benedicks \& Carleson and others, it has been known that a positive measure set of quadratic maps admit invariant probability measures absolutely continuous with respect to Lebesgue. These measures allow one to statistically predict the asymptotic fate of Lebesgue almost every initial condition. Estimating fluctuations of empirical distributions before they settle to equilibrium requires a fairly good control over large parts of the phase space. We use the sub-exponential slow recurrence condition of Benedicks \& Carleson to build induced Markov maps of arbitrarily small scale and associated towers, to which the absolutely continuous measures can be lifted. These various lifts together enable us to obtain a control of recurrence that is sufficient to establish a level 2 large deviation principle, for the absolutely continuous measures. This result encompasses dynamics far from equilibrium, and thus significantly extends presently known local large deviations results for quadratic maps.
\end{abstract}

\section{INTRODUCTION}

Let $X=[-1,1]$, and let $f_{a}: X \circlearrowleft$ be the quadratic map given by $f_{a} x=1-a x^{2}$, where $0<a \leq 2$. The abundance of parameters in this family for which "chaotic dynamics" occur has been known since the pioneering works of Jakobson [20] and Benedicks \& Carleson [5, 6]: there exists a set of $a$-values near 2 with positive Lebesgue measure for which the corresponding $f=f_{a}$ admits an invariant probability measure $\mu$ that is absolutely continuous with respect to Lebesgue (acip). By a classical theorem, for Lebesgue a.e. $x$ the empirical distribution $\delta_{x}^{n}=(1 / n) \sum_{i=0}^{n-1} \delta_{f^{i} x}$ converges weakly to $\mu$. The theory of large deviations aims to provide exponential bounds on the probability that $\delta_{x}^{n}$ stays away from $\mu$.

Large deviations questions have been addressed for various stochastic processes (see e.g. [17, 18]). For dynamical systems, one cannot expect a full large deviation principle without strong assumptions [15, 22, 28, 29, 32, 33, 36. For the quadratic map, the enemy is the critical point $x=0$. Up to now, only local large deviations results are known [21, 24, 30], and a full result which encompasses dynamics far from equilibrium is still unknown. Our aim here is to provide a simple set of conditions satisfied on a positive measure set in parameter space and to show that when these conditions are met, a full large deviation principle holds.

We formulate our conditions as follows: Let $\lambda=\frac{9}{10} \log 2$ and $\alpha=\frac{1}{100}$.

(A1) $f=f_{a}$ where $a$ is sufficiently near 2 ;

(A2) $\left|D f^{n}(f 0)\right| \geq e^{\lambda n}$ for every $n \geq 0$;

(A3) $\left|f^{n} 0\right| \geq e^{-\alpha \sqrt{n}}$ for every $n \geq 1$;

(A4) $f$ is topologically mixing on $\left[f^{2} 0, f 0\right]$.

Benedicks \& Carleson [6] proved the the abundance of parameters near 2 for which (A2) holds. The abundance of parameters for which (A3) holds was proved by Benedicks \& Young [7], and previously by Benedicks \& Carleson [5] under slightly different hypotheses. For their 
parameters, (A4) holds (see [37, Lem. 2.1]). The parameter sets they constructed have 2 as a full Lebesgue density point. Hence, given $a_{0}<2$ arbitrarily near 2 , there is a set $A \subset\left[a_{0}, 2\right]$ with positive Lebesgue measure such that (A2)-(A4) hold for all $a \in A$.

In what follows, we assume (A1)-(A4) for $f=f_{a}$. Then $f$ admits an acip $\mu$. Let $\mathcal{M}$ denote the space of Borel probability measures on $X$ endowed with the topology of weak convergence. Let $\mathcal{M}_{f}$ denote the set of $f$-invariant Borel probability measures. Define a Lyapunov exponent of $\nu \in \mathcal{M}_{f}$ by $\lambda(\nu)=\int \log |D f| d \nu$. This is strictly positive for any $\nu \in \mathcal{M}_{f}$ [9, 27]. Let $h(\nu)$ denote the entropy of $\nu$, and define a free energy function $F: \mathcal{M} \rightarrow \mathbb{R} \cup\{-\infty\}$ by

$$
F(\nu)= \begin{cases}h(\nu)-\lambda(\nu) & \text { if } \nu \in \mathcal{M}_{f} ; \\ -\infty & \text { otherwise }\end{cases}
$$

By Ruelle's inequality [31], $F(\nu) \leq 0$ and the equality holds only if $\nu=\mu$ [23]. It is known [9] that the Lyapunov exponent is not lower semi-continuous, and so $-F$ may not be lower semi-continuous. Hence we introduce its lower-semi-continuous regularization $I: \mathcal{M} \rightarrow[0, \infty]$ by

$$
I(\nu)=-\inf _{\mathcal{G}} \sup \{F(\xi): \xi \in \mathcal{G}\}
$$

where the infimum is taken over all neighborhoods $\mathcal{G}$ of $\nu$ in $\mathcal{M}$. Let $\delta_{x}^{n}=(1 / n) \sum_{i=0}^{n-1} \delta_{f^{i} x}$, where $\delta_{f^{i} x}$ is the Dirac measure at $f^{i} x$. Let $\log 0=-\infty$.

Theorem. Let $f=f_{a}$ satisfy (A1)-(A4). Then the large deviation principle holds for $(f, \mu)$ with $I$ the rate function, namely

$$
\varliminf_{n \rightarrow \infty} \frac{1}{n} \log \mu\left\{x \in X: \delta_{x}^{n} \in \mathcal{G}\right\} \geq-\inf \{I(\nu): \nu \in \mathcal{G}\}
$$

for any open set $\mathcal{G} \subset \mathcal{M}$, and

$$
\varlimsup_{n \rightarrow \infty} \frac{1}{n} \log \mu\left\{x \in X: \delta_{x}^{n} \in \mathcal{K}\right\} \leq-\inf \{I(\nu): \nu \in \mathcal{K}\}
$$

for any closed set $\mathcal{K} \subset \mathcal{M}$.

We state a corollary which follows from the Contraction Principle in large deviations, and use it to compare our result with the previous related ones. Let $C(X)$ denote the space of all continuous functions on $X$. For $\varphi \in C(X)$, write $S_{n} \varphi=\sum_{i=0}^{n-1} \varphi \circ f^{i}$ and define

$$
c_{\varphi}=\inf _{x \in X} \lim _{n \rightarrow \infty} \frac{1}{n} S_{n} \varphi(x) \text { and } d_{\varphi}=\sup _{x \in X} \varlimsup_{n \rightarrow \infty} \frac{1}{n} S_{n} \varphi(x) .
$$

The compactness of $\mathcal{M}_{f}$ implies $c_{\varphi}=\min \left\{\nu(\varphi): \nu \in \mathcal{M}_{f}\right\}$ and $d_{\varphi}=\max \left\{\nu(\varphi): \nu \in \mathcal{M}_{f}\right\}$, where $\nu(\varphi)=\int \varphi d \nu$. We assume $c_{\varphi}<d_{\varphi}$, for otherwise it is meaningless to consider $\varphi$. Define $F_{\varphi}:\left[c_{\varphi}, d_{\varphi}\right] \rightarrow \mathbb{R}$ by

$$
F_{\varphi}(t)=\sup \left\{F(\nu): \nu \in \mathcal{M}_{f}, \nu(\varphi)=t\right\}
$$

which is concave and so continuous on $\left(c_{\varphi}, d_{\varphi}\right)$.

Corollary 1. For all $a, b \in\left[c_{\varphi}, d_{\varphi}\right]$ such that $a<b$ we have

$$
\lim _{n \rightarrow \infty} \frac{1}{n} \log \mu\left\{a \leq \frac{1}{n} S_{n} \varphi \leq b\right\}=\max _{a \leq t \leq b} F_{\varphi}(t) .
$$


Our theorem is the first full large deviations result for a positive measure set of quadratic maps, despite a large number of papers over the past thirty years dedicated to stochastic properties of chaotic dynamics in one-dimensional maps. Up to now, only local results are known, which claim the existence of the above limit in the case where $\varphi$ is Hölder continuous and $a, b$ are near the mean $\mu(\varphi)[21,24,30]$.

The next corollary follows from Varadhan's integral lemma [16, p.137] and the convex duality of the Fenchel-Legendre transforms [16, p.152].

Corollary 2. For any $\varphi \in C(X)$, the limit

$$
P(\varphi)=\lim _{n \rightarrow \infty} \frac{1}{n} \log \mu\left(e^{S_{n} \varphi}\right)
$$

exists. In addition, $(P, I)$ form a Legendre pair, namely the following holds:

$$
\begin{aligned}
& P(\varphi)=\max \left\{\nu(\varphi)-I(\nu): \nu \in \mathcal{M}_{f}\right\} \text { for } \varphi \in C(X) ; \\
& I(\nu)=\max \{\nu(\varphi)-P(\varphi): \varphi \in C(X)\} \text { for } \nu \in \mathcal{M}_{f} .
\end{aligned}
$$

For a broad class of nonuniformly hyperbolic systems including the quadratic maps we treat here, towers (or inducing schemes) have been heavily used to draw their interesting properties (see e.g. [3, 9, 38, 39]). A proof of the theorem also relies on the construction of induced Markov maps and associated towers. There is a great deal of freedom and flexibility in the construction of towers, and hence the issue is to construct a "nice tower" that captures relevant information of the underlying system. This issue has been addressed in the literature, and it is sometimes referred to as the liftability problem. A less emphasized issue deals with the construction of a family of towers for a given single system, so that they altogether provide relevant information. This type of approach can be found, for instance, in [11, 19, 29], and appears to be successful when it is difficult to obtain necessary information just by considering a single tower.

For our quadratic maps, towers have already been constructed (see e.g. [3, 10, 37, 38]), for which the decay rate of the tail of return times is exponential. We emphasize that exponential tails do not necessarily imply the full large deviation principle, primarily because probabilities of rare events included in the tails are unaccounted for. For instance, for certain Markov processes it is well known [4, 17, 18] that exponential tails of return times are in general not sufficient to ensure a full large deviation principle. They only imply a local large deviation result, which is similar to the results in [24, 30]. A full large deviation principle for stationary processes has been established under very strong mixing conditions [12, 13], which cannot be expected for dynamical systems.

In [14], sufficient conditions on the "shape" of towers were introduced to ensure a full large deviation principle for Lebesgue measure. However, for our quadratic maps it is difficult to construct such "ideal towers", apart from very special cases (e.g. Misiurewicz maps). Therefore, we abandon working with a single tower and instead construct various induced Markov maps and associated towers. We use them together to obtain an upper exponential bound, on the probability that time averages of continuous functions stay away from their spatial averages. We establish the large deviation principle by comparing this upper bound with a lower exponential one, which is obtained directly from [14, 26, 37]. The upper and lower large deviation bounds were obtained in a very general setting in [36], and for nonuniformly expanding maps in [1, 34]. These bounds are not comparable and hence insufficient to conclude the large deviation principle. 
A proof of the theorem is briefly outlined as follows. Given $d \geq 1$, functions $\varphi_{1}, \ldots, \varphi_{d}$ on $X$ and $b_{1}, \ldots, b_{d} \in \mathbb{R}$, define

$$
\bar{R}\left(\varphi_{1}, \ldots, \varphi_{d} ; b_{1}, \ldots, b_{d}\right)=\varlimsup_{n \rightarrow \infty} \frac{1}{n} \log \mu\left\{\frac{1}{n} S_{n} \varphi_{j} \geq b_{j}, \quad j=1, \ldots, d\right\}
$$

and

$$
\underline{R}\left(\varphi_{1}, \ldots, \varphi_{d} ; b_{1}, \ldots, b_{d}\right)=\varliminf_{n \rightarrow \infty} \frac{1}{n} \log \mu\left\{\frac{1}{n} S_{n} \varphi_{j}>b_{j}, \quad j=1, \ldots, d\right\} .
$$

All our effort is dedicated to proving the following proposition.

Proposition. Let $f=f_{a}$ satisfy (A1)-(A4). Let $d \geq 1$ and let $\varphi_{1}, \ldots, \varphi_{d}$ be a collection of Lipschitz continuous functions on $X$, and let $b_{1}, \ldots, b_{d} \in \mathbb{R}$. For any $\varepsilon>0$ there exists $n_{0} \in \mathbb{N}$ such that if $n \geq n_{0}$ then there exists $\sigma \in \mathcal{M}_{f}$ such that:

$$
\begin{gathered}
\frac{1}{n} \log \mu\left\{\frac{1}{n} S_{n} \varphi_{j} \geq b_{j}, j=1, \ldots, d\right\} \leq\left(1-\varepsilon^{1 / 5}\right) F(\sigma)+2 \varepsilon^{1 / 5} ; \\
\sigma\left(\varphi_{j}\right) \geq b_{j}-\varepsilon^{1 / 2}, \quad j=1, \ldots, d .
\end{gathered}
$$

It then follows that

$$
\bar{R}\left(\varphi_{1}, \ldots, \varphi_{d} ; b_{1}, \ldots, b_{d}\right) \leq \lim _{\varepsilon \rightarrow 0} \sup \left\{F(\nu): \nu \in \mathcal{M}_{f}, \nu\left(\varphi_{j}\right) \geq b_{j}-\varepsilon^{1 / 2}, \quad j=1, \ldots, d\right\},
$$

where we let $\sup \emptyset=-\infty$ by convention. Meanwhile, by a result of [26, 37], the density of $\mu$ is uniformly bounded away from zero on $\left[f^{2} 0, f 0\right]$. Hence, the lower bound obtained in [14] for Lebesgue translates into a lower bound for $\mu$, namely

$$
\underline{R}\left(\varphi_{1}, \ldots, \varphi_{d} ; b_{1}, \ldots, b_{d}\right) \geq \sup \left\{F(\nu): \nu \in \mathcal{M}, \nu\left(\varphi_{j}\right)>b_{j}, \quad j=1, \ldots, d\right\} .
$$

Observe that the weak topology on $\mathcal{M}$ has a countable base generated by open sets of the form $\left\{\nu \in \mathcal{M}: \nu\left(\varphi_{j}\right)>b_{j}, j=1, \ldots, d\right\}$, where $d \geq 1$, each $\varphi_{j}$ is Lipschitz continuous and $b_{j} \in \mathbb{R}$. Hence, (3) (4) imply the theorem.

Our strategy for the proof of the proposition is to construct a family of towers and use them to construct various horseshoes carrying invariant measures with the properties as in the statement. At this point, we make important use of the sub-exponential slow recurrence condition (A3).

The rest of this paper consists of two sections. In Sect. 2 we develop preliminary estimates and constructions. We modify the classical binding argument and the return time estimate [5. 6], so that we can treat an arbitrarily small $\varepsilon$. In Sect. 3 we prove the proposition. A crucial estimate is Lemma 3.11, which roughly states that any partition element of the tower is approximated by points which quickly return to the base of the tower. To equip our tower with this property, we construct an induced map on a Cantor set, which consists of points slow recurrent to the critical point. This construction is inspired by that of Benedicks \& Young for Hénon-like attractors 8].

To maintain the brevity of this paper we refrain from generalizations. Our arguments and results may be generalized to $C^{2}$ Collet-Eckmann unimodal maps with non-flat critical point, for which the recurrence of the critical orbit is sub-exponential. It is known [2] that almost every stochastic quadratic map satisfies these two conditions. 


\section{Preliminary estimates and constructions}

In this section, we develop preliminary estimates needed for the proof of the proposition. We develop a binding argument for recovering expansion and prove a return time estimate. Original ideas for these can be found in [5, 6]. We modify them to treat an arbitrarily small $\varepsilon>0$. We suppose that $\varepsilon$ is given, and then choose sufficiently large integer $N$.

We use the following notations: $c_{0}=f 0$ and $c_{n}=f^{n} c_{0}$ for $n \geq 1 ;|\cdot|$ is the Lebesgue measure on $X$; for a set $A \subset X, d(0, A)=\inf \{|x|: x \in A\}$; given a partition $\mathcal{P}$ of $A \subset X$ and $B \subset A, \mathcal{P} \mid B=\{\omega \cap B: \omega \in \mathcal{P}\}$.

2.1. Bounded distortion. For $n \geq 1$, let

$$
D_{n}=\frac{1}{10} \cdot\left[\sum_{i=0}^{n-1} d_{i}^{-1}\right]^{-1}, \quad \text { where } \quad d_{i}=\frac{\left|c_{i}\right|}{\left|D f^{i}\left(c_{0}\right)\right|} .
$$

Lemma 2.1. For all $x, y \in I=\left[1-D_{n}, 1\right]$,

$$
\frac{D f^{n}(x)}{D f^{n}(y)} \leq 2 \text { and }\left|\frac{D f^{n}(x)}{D f^{n}(y)}-1\right| \leq \frac{|x-y|}{D_{n}} .
$$

Proof. The first inequality would hold if for every $0 \leq j \leq n-1$ we have

$$
0 \notin f^{j} I, \quad \frac{\left|f^{j} I\right|}{d\left(0, f^{j} I\right)} \leq \log 2 \cdot d_{j}^{-1}\left[\sum_{i=0}^{n-1} d_{i}^{-1}\right]^{-1} .
$$

Indeed, if this is the case, then for $x, y \in I$,

$$
\log \frac{D f^{n}(x)}{D f^{n}(y)} \leq \sum_{j=0}^{n-1} \log \frac{D f\left(f^{j} x\right)}{D f\left(f^{j} y\right)} \leq \sum_{j=0}^{n-1} \frac{\left|f^{j} I\right|}{d\left(0, f^{j} I\right)} \leq \log 2 .
$$

The second inequality follows from $|D f(x)|=2 a|x|$ and $\left|D^{2} f(x)\right|=2 a$.

It is immediate to check ([6) for $j=0$. The rest of the proof is by induction on $j$. Let $k>0$ and assume (6) for every $0 \leq j<k$. Summing (6) over all $0 \leq j<k$ implies $\left|D f^{k}(x)\right| \leq 2\left|D f^{k}(y)\right|$ for all $x, y \in I$. Hence

$$
\left|f^{k} I\right| \leq 2\left|D f^{k}\left(c_{0}\right)\right| D_{n}=2\left|f^{k} c_{0}\right| d_{k}^{-1} D_{n} \leq(1 / 5)\left|c_{k}\right|,
$$

and thus $0 \notin f^{k} I$ holds. For the second half of (6] $)$ we have

$$
\frac{\left|f^{k} I\right|}{d\left(0, f^{k} I\right)} \leq \frac{2\left|D f^{k}\left(c_{0}\right)\right| D_{n}}{d\left(0, f^{k} I\right)}=\frac{2 d_{k}^{-1} D_{n} \cdot\left|c_{k}\right|}{d\left(0, f^{k} I\right)} \leq \frac{3}{10} d_{k}^{-1}\left[\sum_{i=0}^{n-1} d_{i}^{-1}\right]^{-1}
$$

For the last inequality we have used $\frac{\left|c_{k}\right|}{d\left(0, f^{k} I\right)} \leq 3 / 2$ which follows from (77).

For $0 \leq i<n$ we have $\frac{\left|c_{i}\right|}{d\left(0, f^{i}[x, y]\right)} \leq 3 / 2$ and $\left|f^{i}[x, y]\right| \leq 2\left|D f^{i}\left(c_{0}\right)\right||x-y| d_{i} d_{i}^{-1}=2 \mid x-$ $y|| c_{i} \mid d_{i}^{-1}$, and thus $\frac{\left|f^{i}[x, y]\right|}{d\left(0, f^{i}[x, y]\right)} \leq 3|x-y| d_{i}^{-1}$. Therefore

$$
\log \frac{D f^{n}(x)}{D f^{n}(y)} \leq \sum_{i=0}^{n-1} \frac{\left|f^{i}[x, y]\right|}{d\left(0, f^{i}[x, y]\right)} \leq \frac{3}{10} \frac{|x-y|}{D_{n}} \leq \frac{3}{10}
$$

The second inequality of the lemma follows from the fact that $e^{z} \leq 1+2 z$ for $0 \leq z \leq 3 / 10$. 
2.2. Recovering expansion. For $p \geq 1$, let $\delta_{p}=\sqrt{e^{-\varepsilon p} D_{p}}$. Here, $\varepsilon>0$ is the small constant in the statement of the proposition. Let $\hat{\delta}=\delta_{10}$. The proof of the next lemma is a slight modification of that of [5, Lem. 1], and hence it is omitted; the next lemma ensures an exponential growth of derivatives outside of $(-\hat{\delta}, \hat{\delta})$.

Lemma 2.2. If $f=f_{a}, x \in X, n \geq 1$ are such that $\left|f^{i} x\right| \geq \hat{\delta}$ for every $0 \leq i \leq n-1$, then $\left|D f^{n}(x)\right| \geq \hat{\delta} e^{\lambda n}$. Moreover, if $\left|f^{n} x\right|<\hat{\delta}$ then $\left|D f^{n}(x)\right| \geq e^{\lambda n}$.

To deal with the loss of expansion due to returns to $(-\hat{\delta}, \hat{\delta})$, we mimic the binding argument of Benedicks \& Carleson [5, 6]: subdivide the interval into pieces, and deal with them independently. Key ingredients are the notion of binding and an associated expansion estimate. We develop them in a slightly different way from [5, 6] for our purpose.

Lemma 2.3. If $p>10$ and $\delta_{p} \leq|x|<\delta_{p-1}$ then:

(a) $\left|D f^{p}(x)\right| \geq e^{\frac{\lambda}{3} p}$;

(b) $p \leq \log |x|^{-\frac{2}{\lambda}}$.

Proof. We have

$$
\begin{aligned}
\left|D f^{p}(x)\right| & =\left|D f^{p-1}(f x)\right||D f(x)| \geq\left|D f^{p-1}\left(c_{0}\right)\right||x| \geq\left|D f^{p-1}\left(c_{0}\right)\right| \delta_{p} \\
& \geq\left|D f^{p-1}\left(c_{0}\right)\right|^{\frac{2}{5}} \geq e^{\frac{\lambda}{3} p}
\end{aligned}
$$

where the first inequality follows from the bounded distortion in Lemma 2.1. For the last two inequalities we have used (A2) and $p>10$. We also have

$$
|x|^{2} \leq D_{p-1} \leq(1 / 10) d_{p-2} \leq(1 / 10)\left|D f^{p-2}\left(c_{0}\right)\right|^{-1} \leq(1 / 10) e^{-\lambda(p-2)} \leq e^{-\lambda p} .
$$

This yields the upper estimate of $p$.

In the following two lemmas, for $x \in X$ we consider a sequence

$$
0 \leq n_{1}(x)<n_{1}(x)+p_{1}(x) \leq n_{2}(x)<n_{2}(x)+p_{2}(x) \leq \cdots
$$

of integers that is defined as follows: $n_{1}=\min \left\{n \geq 0:\left|f^{n} x\right|<\hat{\delta}\right\}$. Given $n_{k}$, define $p_{k}, n_{k+1}$ by $\delta_{p_{k}} \leq\left|f^{n_{k}} x\right|<\delta_{p_{k}-1}$ and $n_{k+1}=\left\{n \geq n_{k}+p_{k}:\left|f^{n} x\right|<\hat{\delta}\right\}$. Lemma 2.2 and Lemma 2.3)(a) yield

$$
\left|D f^{n_{k+1}-n_{k}-p_{k}}\left(f^{n_{k}+p_{k}} x\right)\right| \geq e^{\lambda\left(n_{k+1}-n_{k}-p_{k}\right)} \text { and }\left|D f^{p_{k}}\left(f^{n_{k}} x\right)\right| \geq e^{\frac{\lambda}{3} p_{k}} .
$$

Lemma 2.4. For all $0 \leq i<j,\left|D f^{j-i}\left(c_{i}\right)\right| \geq e^{-\alpha \sqrt{j}}$.

Proof. Fix an integer $M$ such that $\hat{\delta} e^{\alpha \sqrt{M}} \geq 1$. Fix $a_{0}$ sufficiently near 2 such that $\left|D f\left(c_{i}\right)\right| \geq$ 3.5 for every $0 \leq i<M$, and the conclusion of Lemma 2.2 holds for all $a \in\left[a_{0}, 2\right]$. We first consider the case where $\left|c_{n}\right| \geq \hat{\delta}$ for every $i \leq n \leq j-1$. If $j \leq M$, then the choice of $a_{0}$ ensures $\left|D f^{j-i}\left(c_{i}\right)\right| \geq(3.5)^{j-i}$, which is stronger than what is asserted. If $j>M$, then by Lemma 2.2 and $\sqrt{j-i} \geq \sqrt{j}-\sqrt{i}$,

$$
\left|D f^{j-i}\left(c_{i}\right)\right| \geq \hat{\delta} e^{\lambda(j-i)} \geq \hat{\delta} e^{\alpha \sqrt{j-i}} \geq \hat{\delta} e^{\alpha \sqrt{j}-\alpha \sqrt{i}} \geq \hat{\delta} e^{\alpha \sqrt{M}-\alpha \sqrt{i}} \geq e^{-\alpha \sqrt{i}} .
$$

In the case where $\left|c_{n}\right|<\hat{\delta}$ for some $i \leq n \leq j-1$, consider the sequence $\left\{n_{k}, p_{k}\right\}_{k \geq 1}$ for the orbit of $c_{i}$. If $n_{k}+p_{k} \leq j \leq n_{k+1}$ for some $k$, then (8) yields $\left|D f^{j-i}\left(c_{i}\right)\right| \geq \hat{\delta} e^{\frac{\lambda}{3}(j-i)}$, which is 
$\geq e^{-\alpha \sqrt{i}}$ as proved in the first case. If $n_{k}+1 \leq j \leq n_{k}+p_{k}$ for some $k$, then we have

$$
\begin{aligned}
\left|D f^{j-i}\left(c_{i}\right)\right| & =\left|D f^{n_{k}-i}\left(c_{i}\right)\right| \cdot\left|D f\left(c_{n_{k}}\right)\right| \cdot\left|D f^{j-n_{k}-1}\left(c_{n_{k}+1}\right)\right| \\
& \geq e^{\frac{\lambda}{3}\left(n_{k}-i\right)} \cdot 2 a\left|c_{n_{k}}\right| \cdot 2^{-1} e^{\lambda\left(j-n_{k}\right)} \geq e^{\frac{\lambda}{3}(j-i)-\alpha \sqrt{n_{k}}} \geq e^{-\alpha \sqrt{j}} .
\end{aligned}
$$

Let $N$ be a large integer, and set $\delta=\delta_{N} \ll \hat{\delta}$. The next lemma on the growth of derivatives outside of $(-\delta, \delta)$ will be used to construct induced maps with arbitrarily small scale.

Lemma 2.5. The following holds for all sufficiently large $N$ : if $x \in X, n \geq 1$ are such that $\left|f^{i} x\right| \geq \delta$ for every $0 \leq i \leq n-1$, then $\left|D f^{n}(x)\right| \geq \delta e^{\frac{\lambda}{3} n}$. Moreover, if $\left|f^{n} x\right|<\delta$ then $\left|D f^{n}(x)\right| \geq e^{\frac{\lambda}{3} n}$.

Proof. For the orbit of $x$ consider the sequence $\left\{n_{k}, p_{k}\right\}_{k \geq 1}$ and let $s$ be such that $n_{s} \leq n<$ $n_{s+1}$. (8) yields $\left|D f^{n_{s}}(x)\right| \geq e^{\frac{\lambda}{3} n_{s}}$. If $n_{s}+p_{s}>n$, then Lemma 2.1 yields $\left|D f^{n-n_{s}}\left(f^{n_{s}} x\right)\right| \geq$ $(1 / 2)\left|D f\left(f^{n_{s}} x\right)\right|\left|D f^{n-n_{s}-1}\left(c_{0}\right)\right| \geq a \delta e^{\lambda\left(n-n_{s}-1\right)} \geq \delta e^{\frac{\lambda}{3}\left(n-n_{s}\right)}$. If $n_{s}+p_{s} \leq n$, then Lemma 2.2 yields $\left|D f^{n-n_{s}}\left(f^{n_{s}} x\right)\right| \geq \hat{\delta} e^{\frac{\lambda}{3}\left(n-n_{s}\right)}$. Hence the first estimate of Lemma 2.5 holds.

Lemma 2.1 and the definition (15) yield $\left|f^{i} x-c_{i-n_{s}-1}\right| \leq(1 / 5)\left|c_{i-n_{s}-1}\right|$ for every $n_{s} \leq i \leq$ $n_{s}+p_{s}$, and thus $\left|f^{i} x\right| \geq(4 / 5)\left|c_{i-n_{s}-1}\right| \geq(4 / 5) e^{-\alpha \sqrt{p_{s}}} \geq(4 / 5) e^{-\alpha \sqrt{\frac{2}{\lambda} \log \left|f^{n_{s}} x\right|}}>\left|f^{n_{s}} x\right| \geq \delta$. We have used (A3) for the second inequality and Lemma 2.3 (b) for the third. The fourth inequality holds because $\left|f^{n_{s}} x\right|<\hat{\delta} \ll 1$. Hence, if $\left|f^{n} x\right|<\delta$ then $n_{s}+p_{s} \leq n$, and so the factor $\hat{\delta}$ above can be dropped by Lemma 2.2 .

2.3. Inducing to small scales. For each $p>N$, divide the interval $\left[\delta_{p}, \delta_{p-1}\right)$ into $\left[e^{3 \varepsilon p}\right]$ number of subintervals of equal length and denote them by $I_{p, j}\left(j=1,2, \ldots,\left[e^{3 \varepsilon p}\right]\right)$, from the right to the left. Let $I_{p,-j}=-I_{p, j}$, which is the mirror image of $I_{p, j}$ with respect to 0 .

Lemma 2.6. If $N$ is sufficiently large, then for every $I_{p, j}$ the following holds:

(a) $\left|f^{p} I_{p, j}\right| \geq e^{-5 \varepsilon p}$;

(b) $\left|I_{p, j}\right| \leq d\left(0, I_{p, j}\right)^{1+\frac{\varepsilon}{3}}$;

(c) for all $x, y \in I_{p, j}, \log \frac{D f^{p}(x)}{D f^{p}(y)} \leq\left|f^{p} x-f^{p} y\right|^{\varepsilon^{2}}$.

Proof. We have

$$
\left|f I_{p, j}\right| \geq e^{-3 \varepsilon p}\left|\delta_{p-1}-\delta_{p}\right| \delta_{p} \geq e^{-4 \varepsilon p}\left(e^{\frac{\varepsilon}{2}}-1\right) D_{p}
$$

and thus

$$
\left|f^{p} I_{p, j}\right| \geq(1 / 2)\left|D f^{p-1}\left(c_{0}\right)\right|\left|f I_{p, j}\right| \geq e^{-4 \varepsilon p}\left(e^{\frac{\varepsilon}{2}}-1\right)\left|D f^{p-1}\left(c_{0}\right)\right| D_{p} .
$$

Using (A3) and Lemma 2.4 to estimate the second factor we have

$$
\left|D f^{p-1}\left(c_{0}\right)\right|^{-1} D_{p}^{-1}=\sum_{j=0}^{p-1}\left|c_{j}\right|^{-1} \frac{\left|D f^{j}\left(c_{0}\right)\right|}{\left|D f^{p-1}\left(c_{0}\right)\right|} \leq p e^{2 \alpha \sqrt{p}} \leq e^{3 \alpha \sqrt{p}} .
$$

Taking reciprocals and plugging the result into the above inequality,

$$
\left|f^{p} I_{p, j}\right| \geq\left(e^{\frac{\varepsilon}{2}}-1\right) e^{-4 \varepsilon p-3 \alpha \sqrt{p}} \geq e^{-5 \varepsilon p} .
$$

Hence (a) holds. 
Using (A3) we have

$$
\frac{\delta_{p-1}^{2}}{\delta_{p}^{2}} \leq e^{\varepsilon}\left(1+\frac{d_{p-1}}{d_{p}}\right)=e^{\varepsilon}\left(1+\frac{\left|c_{p-1}\right|}{\left|D f\left(c_{p-1}\right)\right|\left|c_{p}\right|}\right) \leq 3 e^{\alpha \sqrt{p}} .
$$

Hence $\left|I_{p}\right| \leq \delta_{p-1} \leq \delta_{p} \sqrt{3 e^{\alpha \sqrt{p}}}$, and thus $\left|I_{p, j}\right| \leq e^{-3 \varepsilon p}\left|I_{p}\right| \leq e^{-\varepsilon p} \delta_{p}$. Since $e^{-\varepsilon p} \leq 10^{-\frac{\varepsilon p}{3}} \leq \delta_{p}^{\frac{\varepsilon}{3}}$ we have $\left|I_{p, j}\right| \leq e^{-\varepsilon p} \delta_{p} \leq \delta_{p}^{1+\frac{\varepsilon}{3}} \leq d\left(0, I_{p, j}\right)^{1+\frac{\varepsilon}{3}}$, and (b) holds.

We have $\left|D f^{p}(x)-D f^{p}(y)\right| \leq I+I I$, where

$$
I=\left|D f^{p-1}(f x)\right||D f(x)-D f(y)|, \quad I I=|D f(y)|\left|D f^{p-1}(f y)\right| \frac{D f^{p-1}(f x)}{D f^{p-1}(f y)}-1 \mid .
$$

By (b),

$$
I \leq 8\left|D f^{p-1}\left(c_{0}\right)\right||x-y| \leq 8\left|D f^{p-1}\left(c_{0}\right)\right| d\left(0, I_{p, j}\right)|x-y|^{\frac{\varepsilon}{3+\varepsilon}} .
$$

The second inequality of Lemma 2.1 gives

$$
\left|\frac{D f^{p-1}(f x)}{D f^{p-1}(f y)}-1\right| \leq \frac{|x-y|^{2}}{D_{p-1}} \leq \frac{|x-y|^{2}}{d\left(0, I_{p, j}\right)^{2}} \leq|x-y|^{\frac{2 \varepsilon}{3+\varepsilon}}
$$

Using this and $|D f(y)| \leq 2 \cdot d\left(0, I_{p, j}\right)$ which follows from (b) we get

$$
I I \leq 4 d\left(0, I_{p, j}\right)\left|D f^{p-1}\left(c_{0}\right)\right||x-y|^{\frac{2 \varepsilon}{3+\varepsilon}} .
$$

Combining (10) (11) with $\left|D f^{p}(y)\right| \geq\left|D f^{p-1}\left(c_{0}\right)\right| d\left(0, I_{p, j}\right)$ yields

$$
\left|\frac{D f^{p}(x)}{D f^{p}(y)}-1\right| \leq 8|x-y|^{\frac{\varepsilon}{3+\varepsilon}} \leq\left|f^{p} x-f^{p} y\right|^{\frac{\varepsilon}{3+\varepsilon}} \leq\left|f^{p} x-f^{p} y\right|^{\varepsilon^{2}}
$$

which implies (c). The last inequality is because $\left|f^{p} x-f^{p} y\right|<1$ and $\frac{\varepsilon}{3+\varepsilon}>\varepsilon^{2}$.

2.4. Combinatorics of partitions. Let $\Lambda^{+}=I_{N, 1}$ (the right extremal $I_{p, j^{-}}$interval), $\Lambda^{-}=$ $-\Lambda^{+}$and $\Lambda=\Lambda^{-} \cup \Lambda^{+}$. By induction on the number of iterations we construct a "decreasing" sequence $\left\{\tilde{\mathcal{P}}_{n}\right\}_{n=0}^{\infty}$ of partitions of $\Lambda$ into intervals, and introduce the notion of bound/free states. Start with $\tilde{\mathcal{P}}_{0}=\left\{\Lambda^{+}, \Lambda^{-}\right\}$. We refer to $\Lambda^{ \pm}$and $f^{N} \Lambda^{ \pm}$as free and to $f^{i} \Lambda^{ \pm}(1 \leq i \leq$ $N-1)$ as bound. Call $p_{0}\left(\Lambda^{ \pm}\right)=N$ a bound period of $\Lambda^{ \pm}$at time 0 .

Set $\tilde{\mathcal{P}}_{0}=\tilde{\mathcal{P}}_{1}=\cdots=\tilde{\mathcal{P}}_{N-1}$, and let $n \geq N$. The $f^{n}$-images of elements of $\tilde{\mathcal{P}}_{n-1}$ are in two phases: either bound or free. If $\omega \in \tilde{\mathcal{P}}_{n-1}, f^{n} \omega$ is free and $d\left(0, f^{n} \omega\right)<\delta$, then $\tilde{\mathcal{P}}_{n}$ subdivides $\omega$. For each resulting element $\omega^{\prime} \in \tilde{\mathcal{P}}_{n} \mid \omega$ with $d\left(0, f^{n} \omega^{\prime}\right)<\delta$ an integer $p_{n}\left(\omega^{\prime}\right)$ is attached; this integer is called a bound period of $\omega^{\prime}$ at time $n$. We say $n$ is a free return time of $\omega^{\prime}$.

Given $\omega \in \tilde{\mathcal{P}}_{n-1}, \tilde{\mathcal{P}}_{n} \mid \omega$ is defined as follows. If $f^{n} \omega$ is free and contains at least two $I_{p, j^{-}}$ intervals, then let $\tilde{\mathcal{P}}_{n}$ subdivide $\omega$ according to the $(p, j)$-locations of its $f^{n}$-image. In all other cases, let $\tilde{\mathcal{P}}_{n} \mid \omega=\{\omega\}$. Partition points are inserted only to ensure that the $f^{n}$-images of $\tilde{\mathcal{P}}_{n}$-elements intersecting $(-\delta, \delta)$ contain exactly one $I_{p, j} \cdot f^{n}$-images out of $(-\delta, \delta)$ are treated as follows. Let $\omega^{\prime} \subset \omega$ be such that $f^{n} \omega^{\prime}$ is a component of $f^{n} \omega \backslash(-\delta, \delta)$. We let $\omega^{\prime} \in \tilde{\mathcal{P}}_{n}$ if $\left|f^{n} \omega^{\prime}\right| \geq\left|\Lambda^{+}\right|$. Otherwise, we glue $\omega^{\prime}$ to the adjacent element whose $f^{n}$-image contains $\Lambda^{ \pm}$.

The bound periods at time $n$ of the elements of $\tilde{\mathcal{P}}_{n} \mid \omega$ are determined by the $p$-locations of their $f^{n}$-images. Namely, if $\tilde{\mathcal{P}}_{n}$ subdivides $\omega, \omega^{\prime} \in \tilde{\mathcal{P}}_{n} \mid \omega$ and $p_{n}\left(\omega^{\prime}\right)$ makes sense, then $p_{n}\left(\omega^{\prime}\right)=p$ where $p$ is such that $f^{n} \omega^{\prime} \supset I_{p, j}$ holds for some $j$. If $\tilde{\mathcal{P}}_{n} \mid \omega=\{\omega\}$ and $p_{n}(\omega)$ makes sense, then $p_{n}(\omega)=\min \left\{p: I_{p} \cap f^{n} \omega \neq \emptyset\right\}$. 
Let $\omega^{\prime} \in \tilde{\mathcal{P}}_{n}$. We say $f^{n+1} \omega^{\prime}$ is bound if there exists $k \leq n$ such that $\omega^{\prime} \in \tilde{\mathcal{P}}_{k}, p_{k}\left(\omega^{\prime}\right)$ makes sense and satisfies $n+1<k+p_{k}\left(\omega^{\prime}\right)$. Otherwise, we say $f^{n} \omega^{\prime}$ is free.

We need a couple of lemmas on the elements of the partitions.

Lemma 2.7. There exist $C_{\varepsilon}>1, C_{\delta}>1$ such that if $\omega \in \tilde{\mathcal{P}}_{n-1}$ and $f^{n} \omega$ is free, then the following holds for all $x, y \in \omega$ :

(a) $\frac{D f^{n}(x)}{D f^{n}(y)}<C_{\delta}$

(b) Moreover, if $f^{n}[x, y] \subset(-\delta, \delta)$, then $\frac{D f^{n}(x)}{D f^{n}(y)} \leq C_{\varepsilon}$.

Proof. Let $n_{1}<\cdots<n_{s}<n$ denote all the free return times in the first $n$-iterates of $\omega$, with $p_{1}, \ldots, p_{s}$ the corresponding bound periods defined as above. We decompose the time interval $\left[n_{j}+p_{j}, n\right]$ into bound and free segments, and then apply Lemma 2.3 to each bound segment and Lemma 2.5 to each free segment. This yields $\left|f^{n_{j}+p_{j}}[x, y]\right| \leq \delta^{-1} e^{-\frac{\lambda}{3}\left(n-n_{j}-p_{j}\right)}\left|f^{n}[x, y]\right|$. If $f^{n}[x, y] \subset(-\delta, \delta)$, then $\delta$ can be dropped by the last assertion of Lemma 2.5,

For each bound segment, using this estimate and Lemma 2.6)(c) we get

$$
\log \frac{D f^{p_{j}}\left(f^{n_{j}} x\right)}{D f^{p_{j}}\left(f^{n_{j}} y\right)} \leq\left|f^{n_{j}+p_{j}}[x, y]\right|^{\varepsilon^{2}} \leq \delta^{-\varepsilon^{2}} e^{-\varepsilon^{2} \frac{\lambda}{3}\left(n-n_{j}-p_{j}\right)} \cdot\left|f^{n}[x, y]\right|^{\varepsilon^{2}} .
$$

Therefore

$$
\sum_{i \in \cup_{j=1}^{s}\left(n_{j}, n_{j}+p_{j}\right)} \log \frac{D f\left(f^{i} x\right)}{D f\left(f^{i} y\right)} \leq \delta^{-\varepsilon^{2}} \sum_{k=1}^{\infty} e^{-\varepsilon^{2} \frac{\lambda}{3} k} \cdot\left|f^{n}[x, y]\right|^{\varepsilon^{2}} .
$$

For free segments we have

$$
\begin{aligned}
\sum_{i \in[0, n) \backslash \cup_{j=1}^{s}\left(n_{j}, n_{j}+p_{j}\right)} \log \frac{D f\left(f^{i} x\right)}{D f\left(f^{i} y\right)} & \leq 4 \delta^{-1} \sum_{i \in[0, n) \backslash \cup_{j=1}^{s}\left(n_{j}, n_{j}+p_{j}\right)}\left|f^{i}[x, y]\right| \\
& \leq 4 \delta^{-2}\left|f^{n}[x, y]\right| \sum_{i=0}^{n-1} e^{-\frac{\lambda}{3}(n-i)}
\end{aligned}
$$

Set $C_{\delta}=\exp \left(\delta^{-3}\right)$. Then (a) holds. If $f^{n}[x, y] \subset(-\delta, \delta)$, then the multiplicative constants $\delta^{-\varepsilon^{2}}$ and $\delta^{-2}$ on the right-hand-sides can be replaced by 1 and $\delta^{-1}$ respectively. Set $C_{\varepsilon}=$ $\exp \left(10 \sum_{k=1}^{\infty} e^{-\varepsilon^{2} \frac{\lambda}{3} k}\right)$. Then (b) holds.

We define inductively a sequence $\mathcal{F}_{1}, \mathcal{F}_{2}, \ldots$ of partitions of a (full measure) subset of $\Lambda$ and a sequence $S_{1}, S_{2}, \ldots$ of stopping time functions for which the following holds for every $k \geq 1$ :

- $\mathcal{F}_{k} \subset \bigcup_{n \geq N} \tilde{\mathcal{P}}_{n}$;

- for each $\omega \in \mathcal{F}_{k}, f^{S_{k}(\omega)} \omega=\Lambda^{+}$or $=\Lambda^{-}$, and $f^{S_{k}(\omega)}$ maps a neighborhood of $\omega$ diffeomorphically onto $3 \Lambda^{+}$or $3 \Lambda^{-}$(the intervals centered at the midpoint of $\Lambda^{ \pm}$and three times its length).

Start with $k=1$. Let $n \geq N$ and $\omega \in \tilde{\mathcal{P}}_{n-1}$. If $f^{n} \omega$ is free and $f^{n} \omega \supset 3 \Lambda^{+}$or $\supset 3 \Lambda^{-}$, then set $\omega^{\prime}=\omega \cap f^{-n} \Lambda^{+}$or $\omega^{\prime}=\omega \cap f^{-n} \Lambda^{-}$, which is an element of $\tilde{\mathcal{P}}_{n}$. Let $\omega^{\prime} \in \mathcal{F}_{1}$ and $S_{1}\left(\omega^{\prime}\right)=n$. Subsequently we iterate the remaining parts $f^{n} \omega \backslash \Lambda^{+}$or $f^{n} \omega \backslash \Lambda^{-}$and repeat the same construction. By Lemma 2.8 below, $\mathcal{F}_{1}, \mathcal{F}_{2}, \ldots$ are partitions of a full measure subset of $\Lambda$. 
Given $\mathcal{F}_{k}, S_{k}$, let $\omega \in \mathcal{F}_{k}$. Without loss of generality we may assume $f^{S_{k}(\omega)} \omega=\Lambda^{+}$. Define $\mathcal{F}_{k+1} \mid \omega$ to be the pull-back of $\mathcal{F}_{1} \mid \Lambda^{+}$under $f^{S_{k}(\omega)} \mid \omega$. For $\omega^{\prime} \in \mathcal{F}_{k+1} \mid \omega$ define $S_{k+1}\left(\omega^{\prime}\right)=$ $S_{k}(\omega)+S_{1}\left(f^{S_{k}(\omega)} \omega^{\prime}\right)$.

2.5. Inducing to large scales. Let $m>0$ and $\omega \in \tilde{\mathcal{P}}_{m-1}$ be such that $f^{m} \omega$ is free. Multiple stopping times can occur in the first $m$-iterates of $\omega$. Define

$$
e(\omega)=\min \left\{k \geq 1: \omega \text { is not contained in an element of } \mathcal{F}_{k}\right\},
$$

and consider the conditional probability

$$
\left|\left\{S_{e(\omega)} \geq m+n \mid \omega\right\}\right|=\frac{1}{|\omega|}\left|\bigcup\left\{\omega^{\prime} \in \mathcal{F}_{e(\omega)} \mid \omega: S_{e(\omega)}\left(\omega^{\prime}\right) \geq m+n\right\}\right| \in[0,1] .
$$

Lemma 2.8. There exist $n_{0}^{\prime}>0, C>0$ and $\zeta \in(0,1)$ such that if $m \geq n_{0}^{\prime}, \omega \in \tilde{\mathcal{P}}_{m-1}$ and $f^{m} \omega$ is free, then $\left|\left\{S_{e(\omega)} \geq m+n \mid \omega\right\}\right| \leq C \zeta^{n}$ for every $n \geq \varepsilon^{1 / 2} m$.

Proof. Let $\mathcal{G}=\left\{\omega^{\prime} \in \mathcal{F}_{e(\omega)} \mid \omega: S_{e(\omega)}\left(\omega^{\prime}\right) \geq m+n\right\}$. Let $\mathcal{G}^{\prime}$ denote the set of all $\omega^{\prime} \in \mathcal{G}$ for which there exists $m \leq k<m+n$ such that $d\left(0, f^{k} \omega_{k}^{\prime}\right)<\delta$ holds for the element $\omega_{k}^{\prime} \in \tilde{\mathcal{P}}_{k}$ containing $\omega^{\prime}$. Let $\mathcal{G}^{\prime \prime}=\mathcal{G} \backslash \mathcal{G}^{\prime}$.

Each $\omega^{\prime} \in \mathcal{G}^{\prime}$ has an itinerary $\left(n_{1}, p_{1}, j_{1}\right), \ldots,\left(n_{s}, p_{s}, j_{s}\right)$ that is defined as follows: $m \leq$ $n_{1}<\cdots<n_{s}<m+n$ is a sequence of integers, associated with a nested sequence $\omega \supset$ $\omega_{n_{1}} \supset \cdots \supset \omega_{n_{s}} \supset \omega^{\prime}$ of intervals such that for each $i, \omega_{n_{i}}$ is the element of $\tilde{\mathcal{P}}_{n_{i}}$ containing $\omega^{\prime}$ that arises out of the subdivision at time $n_{i}$, with $d\left(0, f^{n_{i}} \omega_{n_{i}}\right)<\delta$ and $\left(p_{i}, j_{i}\right)$ its $(p, j)$ location. Let $n_{s+1} \geq m+n$ be such that $\tilde{\mathcal{P}}_{n_{s+1}}$ partitions $\omega_{n_{s}}$. For any $x \in \omega^{\prime}$ we have $\left|D f^{n_{s+1}}(x)\right| \geq \delta e^{\frac{\lambda}{3} \sum_{i=1}^{s} p_{i}}\left|D f^{m}(x)\right|$ and $\left|D f^{m}(x)\right| \geq C_{\delta}^{-1}\left|f^{m} \omega\right| /|\omega|$, and thus $\left|\omega^{\prime}\right| \leq\left|\omega_{n_{s}}\right| \leq$ $C_{\delta} \delta^{-1} e^{-\frac{\lambda}{3} \sum_{i=1}^{s} p_{i}}|\omega| /\left|f^{m} \omega\right|$. Then

$$
\begin{aligned}
\sum_{\omega^{\prime} \in \mathcal{G}^{\prime}}\left|\omega^{\prime}\right| & =\sum_{s} \sum_{P} \sum_{\substack{\left\{\left(n_{i}, p_{i}, j_{i}\right)\right\}_{i=1}^{s} \\
\sum_{i=1}^{s} p_{i}=P}}\left|\omega^{\prime}\right| \\
& \leq C_{\delta} \delta^{-1} \frac{|\omega|}{\left|f^{m} \omega\right|} \sum_{s} \sum_{P} e^{-\frac{\lambda}{3} P} \#\left\{\left\{\left(n_{i}, p_{i}, j_{i}\right)\right\}_{i=1}^{s}: \sum_{i=1}^{s} p_{i}=P\right\} .
\end{aligned}
$$

The integer $s$ in the summand ranges up to $[n / N]$. Since $1 \leq\left|j_{i}\right| \leq e^{3 \varepsilon p_{j}}$, the number of all sequences $\left\{\left(p_{i}, j_{i}\right)\right\}_{i=1}^{s}$ with $\sum_{i=1}^{s} p_{i}=P$ is $\leq 2^{s}\left(\begin{array}{c}P+s \\ s\end{array}\right) e^{3 \varepsilon P}$. Since there are at most $\left(\begin{array}{c}n \\ s\end{array}\right)$ number of ways of distributing $n_{1}, \ldots, n_{s}$ in $[m, m+n)$, by the Stirling formula for factorials we get

$$
\#\left\{\left\{\left(n_{i}, p_{i}, j_{i}\right)\right\}_{i=1}^{s}: \sum_{i=1}^{s} p_{i}=P\right\} \leq\left(\begin{array}{c}
n \\
s
\end{array}\right) 2^{s}\left(\begin{array}{c}
P+s \\
s
\end{array}\right) e^{3 \varepsilon P} \leq e^{\varepsilon n} e^{4 \varepsilon P} .
$$

Sublemma 2.9. For every $1 \leq i \leq s, n_{i+1}-n_{i} \leq 2 p_{i}$.

Proof. Lemma 2.6(a) gives $\left|f^{n_{i}+p_{i}} \omega_{i}\right| \geq e^{-5 \varepsilon p_{i}}$, and thus $n_{i+1}-n_{i}-p_{i} \leq \frac{6 \varepsilon p_{i}}{\lambda}-\log \delta$; for otherwise, Lemma 2.2 would yield $\left|f^{n_{i+1}} \omega_{i}\right|>2$, which is a contradiction. A simple computation shows $-\log \delta<N \leq p_{i}$, and thus $n_{i+1}-n_{i} \leq 2 p_{i}$. 
It follows that $n<n_{s+1} \leq n_{1}+2 \sum_{i=1}^{s} p_{i}$. If $n_{1} \leq m+n / 2$ then $\sum_{i=1}^{s} p_{i} \geq n / 4$, and therefore

$$
\sum_{\substack{\omega^{\prime} \in \mathcal{G}^{\prime} \\ n_{1} \leq m+n / 2}}\left|\omega^{\prime}\right| \leq C_{\delta} \delta^{-1} \frac{n}{N} \sum_{P \geq n / 4} e^{\left(8 \varepsilon-\frac{\lambda}{3}\right) P} \frac{|\omega|}{\left|f^{m} \omega\right|} \leq e^{-\frac{\lambda}{13} n} \frac{|\omega|}{\left|f^{m} \omega\right|}
$$

where the last inequality holds provided $m$ is sufficiently large because $n \geq \varepsilon^{1 / 2} m$.

For those $\omega^{\prime} \in \mathcal{G}^{\prime}$ with $n_{1}>m+n / 2$, a similar reasoning shows

$$
\left|\omega^{\prime}\right| \leq\left|\omega_{n_{1}}\right| \leq C_{\delta} \delta^{-1} e^{-\lambda\left(n_{1}-m\right)} \frac{|\omega|}{\left|f^{m} \omega\right|} \leq C_{\delta} \delta^{-1} e^{-\frac{\lambda n}{2}} \frac{|\omega|}{\left|f^{m} \omega\right|} \leq e^{-\frac{\lambda n}{3}} \frac{|\omega|}{\left|f^{m} \omega\right|},
$$

and therefore

$$
\sum_{\substack{\omega^{\prime} \in \mathcal{G}^{\prime} \\ n_{1}>m+n / 2}}\left|\omega^{\prime}\right| \leq C_{\delta} \delta^{-1} \frac{n}{N} \sum_{P \leq n} e^{4 \varepsilon P+\varepsilon n-\frac{\lambda}{3} n} \frac{|\omega|}{\left|f^{m} \omega\right|} \leq e^{-\frac{\lambda n}{4}} \frac{|\omega|}{\left|f^{m} \omega\right|},
$$

where the last inequality holds provided $m$ is sufficiently large because $n \geq \varepsilon^{1 / 2} m$.

We now treat elements of $\mathcal{G}^{\prime \prime}$. Let $r \geq 0$ denote the integer such that $\omega$ is subdivided at time $m+r$. Since $I_{p j} \supset f^{k} \omega$ holds for some $k<m$ we have $\left|f^{m} \omega\right| \geq \delta e^{-5 \varepsilon m}$. If $r \geq \varepsilon^{1 / 2} m$, then $\left|f^{m+n} \omega\right| \geq \delta e^{-5 \varepsilon m} e^{\lambda \varepsilon^{1 / 2} m}>2=|X|$, which is a contradiction. Hence $r<\varepsilon^{1 / 2} m$, and thus $r<n$.

Let $k \geq m$. Let us say that $\tilde{\omega} \in \tilde{\mathcal{P}}_{k} \mid \omega$ is an escaping component at time $k$ if $\tilde{\omega}$ arises out of subdivision at time $k$ and satisfies $d\left(0, f^{k} \tilde{\omega}\right)=\delta$. Let $\mathcal{E}_{1}$ denote the collection of escaping components at time $r$. If $\mathcal{E}_{1}=\emptyset$, then $\mathcal{G}^{\prime \prime}=\emptyset$. Hence we assume $\mathcal{E}_{1} \neq \emptyset$.

Each $\omega^{\prime} \in \mathcal{G}^{\prime \prime}$ has an itinerary $\left(k_{1}, \epsilon_{1}\right), \ldots,\left(k_{t}, \epsilon_{t}\right)$ that is defined as follows: $m \leq k_{1}<\cdots<$ $k_{t}<m+n$ is a sequence of integers, associated with a nested sequence $\omega \supset \omega_{k_{1}} \supset \cdots \supset$ $\omega_{k_{t}} \supset \omega^{\prime}$ of intervals such that for each $i, \omega_{k_{i}}$ is an escaping component at time $k_{i}$ and $\epsilon_{i}=+$ (resp. $\varepsilon_{i}=-$ ) if $f^{k_{i}} \omega_{k_{i}}$ is at the right (resp. left) of the critical point. Call $t$ the length of the itinerary of $\omega^{\prime}$. Using the previous estimates and the fact that $\omega_{k_{t}}$ is not subdivided up to time $m+n-1$, we have $\left|\omega_{k_{t}}\right| \leq e^{-\lambda n / 2}|\omega| /\left|f^{m} \omega\right|$.

Let $\mathcal{H}=\left\{\omega^{\prime} \in \mathcal{G}^{\prime \prime}\right.$ : The length of the itinerary is $\left.\leq \theta n\right\}$. The number of all itineraries of length $t$ is $\leq\left(\begin{array}{c}n \\ t\end{array}\right)$, and so by the Stirling formula one can choose a small constant $\theta>0$ such that $\# \mathcal{H} \leq e^{\lambda n / 100}$. Then

$$
\sum_{\omega^{\prime} \in \mathcal{H}}\left|\omega^{\prime}\right| \leq \# \mathcal{H} e^{-\lambda n / 2} \frac{|\omega|}{\left|f^{m} \omega\right|} \leq e^{-\frac{\lambda}{3} n} \frac{|\omega|}{\left|f^{m} \omega\right|}
$$

To treat elements in $\mathcal{H}^{\prime}=\left\{\omega^{\prime} \in \mathcal{G}^{\prime \prime}\right.$ : The length of the itinerary is $\left.\geq \theta n\right\}$, for each $t \geq 1$ define a collection $\mathcal{E}_{t}$ of escaping components (at variable times) inductively as follows: each $\omega \in \mathcal{E}_{t}$ is an escaping component at some time, say $k=k(\omega)$. Let $k^{\prime}>k$ denote the time at which $\omega$ is subdivided. Then $\omega$ contains no or at most two escaping components at time $k^{\prime}$. We let them in $\mathcal{E}_{t+1}$. Let $E_{t}=\bigcup_{\omega \in \mathcal{E}_{t}} \omega$. The bounded distortion in Lemma 2.7 implies that there exists $\hat{\zeta} \in(0,1)$ such that for every $t \geq 1$ and $\omega \in \mathcal{E}_{t},\left|\omega \cap \Omega_{t+1}\right| \leq(1-\hat{\zeta})|\omega|$. Hence $\left|E_{t+1}\right| \leq(1-\hat{\zeta})\left|E_{t}\right|$, and thus $\left|E_{t}\right| \leq(1-\hat{\zeta})^{t}|\omega|$. By definition, if the itinerary of $\omega^{\prime} \in \mathcal{H}^{\prime}$ is of length $t$, then $\omega^{\prime}$ is contained in an element of $\mathcal{E}_{t}$. Hence

$$
\sum_{\omega^{\prime} \in \mathcal{H}^{\prime}}\left|\omega^{\prime}\right| \leq \sum_{\theta n \leq t \leq n}\left|E_{t}\right| \leq \sum_{t \geq \theta n}(1-\hat{\zeta})^{t} \leq \hat{\zeta}^{-1}(1-\hat{\zeta})^{\theta n}
$$


Set $C=1+\hat{\zeta}^{-1}$ and $\zeta=\max \left\{e^{-\frac{\lambda}{14}},(1-\hat{\zeta})^{\theta}\right\}$. (13) (14) (15) yield $\left|\left\{S_{e(\omega)} \geq m+n \mid \omega\right\}\right| \leq$ $e^{-\frac{\lambda}{14} n}+\hat{\zeta}^{-1}(1-\hat{\zeta})^{\theta n} \leq C \zeta^{n}$.

\section{Proof OF THE PROPOSITION}

In this last section we prove the proposition. In Sect. 3.1 we construct a Cantor set $\Omega_{\infty}$ of positive Lebesgue measure. In Sect. 3.2 and Sect. 3.3 we construct an induced map $F: \Omega_{\infty} \circlearrowleft$ and then define an associated tower $\Delta$. In Sect. 3.4 we show that this tower has a distinctive property, and in Sect. 3.5 use this property to construct a certain convenient horseshoe. In Sect. 3.6 we construct an invariant probability measure with the properties in the statement of the proposition.

3.1. Construction of a positive measure set. We construct a subset $\Omega_{\infty}$ of $\Lambda$ with positive Lebesgue measure. Let $\Omega_{N-1}=\Lambda$. For $n \geq N$ we inductively define

$$
\Omega_{n}=\Omega_{n-1} \backslash \bigcup\left\{\omega \in \tilde{\mathcal{P}}_{n}: d\left(0, f^{n} \omega\right)<\delta e^{-\varepsilon n}\right\},
$$

and set $\Omega_{\infty}=\bigcap_{n \geq N-1} \Omega_{n}$. Any component of $\Omega_{n-1} \backslash \Omega_{n}$ is called a gap of order $n$.

Lemma 3.1. For any $n \geq 0$ and $\tilde{\omega} \in \tilde{\mathcal{P}}_{n}$ we have $\left|\tilde{\omega} \cap \Omega_{\infty}\right| \geq(1 / 2)|\tilde{\omega}|$. In particular, $\left|\Omega_{\infty}\right| \geq(1 / 2)|\Lambda|$.

Proof. Choose a point $x \in \tilde{\omega} \cap \Omega_{\infty}$. Since $\left|f^{i} x\right| \geq \delta e^{-\varepsilon i}$ for every $N \leq i \leq n$, the element of $\tilde{\mathcal{P}}_{n}$ containing $x$, which is $\tilde{\omega}$, belongs to $\Omega_{n}$. Let $k \geq n+1$ and $\omega \in \tilde{\mathcal{P}}_{k-1} \mid \tilde{\omega}$ be such that $\omega \subset \Omega_{k-1}$, and suppose that some part of it is deleted at step $k$. We claim that $f^{k} \omega$ is free. Indeed, if this is false then for the last free return time $j$ of $\omega$ before $k$ with bound period $p$ we have $k<j+p$. We also have $\left|f^{j+1} \omega\right| \leq 2 \delta_{p}^{2}$, and thus $\left|f^{k} \omega\right| \leq\left|D f^{k-j-1}\left(c_{0}\right)\right| D_{p} \leq(1 / 10)\left|c_{k-j}\right|$. This yields

$$
d\left(0, f^{k} \omega\right) \geq(9 / 10)\left|c_{k-j}\right| \geq(9 / 10) e^{-\alpha \sqrt{p}} \geq(9 / 10) e^{-\alpha \sqrt{\frac{2}{\lambda}(-\log \delta+\varepsilon k)}}>(\delta / 2) e^{-\varepsilon k},
$$

which means that no part of $\omega$ is deleted at step $k$ and a contradiction arises.

Sublemma 3.2. $\left|f^{k} \omega \cap(-\delta, \delta)\right| \geq \delta e^{-\frac{10 \varepsilon^{2}}{\lambda} k}$.

Proof. If $f^{k} \omega \subset(-\delta, \delta)$ then let $i<k$ be such that $f^{i} \omega$ is free and contains some $I_{p, j}$. Since $p \leq \frac{2}{\lambda}(-\log \delta+\varepsilon k)$, Lemma 2.6(a) yields $\left|f^{i+p} \omega\right| \geq e^{-5 \varepsilon p} \geq \delta^{\frac{10 \varepsilon}{\lambda}} e^{-\frac{10 \varepsilon^{2}}{\lambda} k}$. Since $f^{k} \omega$ is free we obtain $\left|f^{k} \omega\right| \geq\left|f^{i+p} \omega\right| \geq \delta e^{-\frac{10 \varepsilon^{2}}{\lambda} k}$. If $f^{k} \omega$ is not contained in $(-\delta, \delta)$, then obviously $\left|f^{k} \omega \cap(-\delta, \delta)\right| \geq \delta\left(1-e^{-\varepsilon k}\right)>\delta e^{-\frac{10 \varepsilon^{2}}{\lambda} k}$.

The subinterval of $f^{k} \omega$ to be deleted has length $\leq 3 \delta e^{-\varepsilon k}$. Taking distortions into consideration when pulling back to $\omega$, we have

$$
\frac{\left|\tilde{\omega} \cap\left(\Omega_{k-1} \backslash \Omega_{k}\right)\right|}{\left|\tilde{\omega} \cap \Omega_{k-1}\right|} \leq 3 C_{\varepsilon} e^{-\left(\varepsilon-\frac{10 \varepsilon^{2}}{\lambda}\right) k} .
$$

This yields

$$
\frac{\left|\tilde{\omega} \cap \Omega_{\infty}\right|}{|\tilde{\omega}|} \geq \prod_{k=N}^{\infty}\left(1-3 C_{\varepsilon} e^{-\left(\varepsilon-\frac{10 \varepsilon^{2}}{\lambda}\right) k}\right) \geq \frac{1}{2}
$$


3.2. Construction of an induced map on the Cantor set. Lebesgue almost every $x \in \Omega_{\infty}$ has an infinite number of stopping times, which we denote by $S_{1}(x)<S_{2}(x)<\cdots$ with a slight abuse of notation. We define its subsequence $R_{1}(x)<R_{2}(x)<\cdots$ and a return time $R(x)$ to $\Omega_{\infty}$ as follows. Start with $R_{1}(x)=S_{1}(x)$. Given $R_{i}(x)$, if $f^{R_{i}(x)} x \in \Omega_{\infty}$ then let $R(x)=R_{i}(x)$. If $f^{R_{i}(x)} x \notin \Omega_{\infty}$, then let $g_{i}$ denote the order of the gap containing $f^{R_{i}(x)} x$ and define $R_{i+1}(x)$ to be the smallest stopping time after $R_{i}(x)+g_{i}$. Note that $R$ is not necessarily the first return time to $\Omega_{\infty}$. The $R_{i}(x)(i=1,2, \ldots)$ are called regular return times of $x \in \Omega_{\infty}$. Let $\Omega_{\infty}^{ \pm}=\Lambda^{ \pm} \cap \Omega_{\infty}$.

Lemma 3.3. There exists a countable partition $\mathcal{Q}$ of a full measure subset of $\Omega_{\infty}$ such that the following holds for every $\omega \in \mathcal{Q}$ :

(a) $R$ is constant on $\omega$ (denote this value by $R(\omega)$ ) and $f^{R(\omega)}$ maps $\omega$ bijectively onto $\Omega_{\infty}^{ \pm}$. In addition,

(b) for all $x, y \in \omega,\left|\frac{D f^{R(\omega)}(x)}{D f^{R(\omega)}(y)}-1\right| \leq 3\left|\Lambda^{+}\right|^{-1}\left|f^{R(\omega)}[x, y]\right|$.

Proof. We construct $\mathcal{Q}$ by induction using the partitions $\mathcal{F}_{k}$ and the stopping times $S_{k}$. To begin with, for all $\omega \in \mathcal{F}_{1}$ intersecting $\Omega_{\infty}$ let $\left(f^{S_{1}(\omega)} \mid \omega\right)^{-1} \Omega_{\infty} \in \mathcal{Q}$.

Sublemma 3.4. If $\omega \in \mathcal{F}_{1}$ and $\omega \cap \Omega_{\infty} \neq \emptyset$, then $\left(f^{S_{1}(\omega)} \mid \omega\right)^{-1} \Omega_{\infty} \subset \Omega_{\infty}$.

Proof. Let $x \in\left(f^{S_{1}(\omega)} \mid \omega\right)^{-1} \Omega_{\infty}$. Since $\omega \cap \Omega_{\infty} \neq \emptyset, \omega \subset \Omega_{S_{1}(\omega)-1}$ holds, and thus $x \in \Omega_{S_{1}(\omega)-1}$. Since $f^{S_{1}(\omega)} x \in \Omega_{\infty}, x \in \Omega_{S_{1}(\omega)}$ holds. Since $f^{S_{1}(\omega)} x=y$ for some $y \in \Omega_{\infty}$, for every $n>0$ we have $\left|f^{n+S_{1}(\omega)} x\right|=\left|f^{n} y\right| \geq \delta e^{-\varepsilon n}>\delta e^{-\varepsilon\left(n+S_{1}(\omega)\right)}$, and thus $x \in \Omega_{n+S_{1}(\omega)}$. This yields $x \in \Omega_{\infty}$.

For $\omega \in \mathcal{F}_{1}$ intersecting $\Omega_{\infty}$, let $G$ be a gap of order $g$ with $G \cap f^{S_{1}(\omega)}\left(\omega \cap \Omega_{\infty}\right) \neq \emptyset$. For any $k>1$ and $\omega^{\prime} \in \mathcal{F}_{k} \mid \omega$ such that: (i) $\omega^{\prime} \cap \Omega_{\infty} \neq \emptyset$; (ii) $f^{S_{1}(\omega)} \omega^{\prime} \subset G$; (iii) $S_{k}\left(\omega^{\prime}\right)=R_{2}(x)$ for $x \in \omega^{\prime} \cap \Omega_{\infty}$, let $\left(f^{S_{k}\left(\omega^{\prime}\right)} \mid \omega^{\prime}\right)^{-1} \Omega_{\infty} \in \mathcal{Q}$.

Sublemma 3.5. $\left(f^{S_{k}(\omega)} \mid \omega^{\prime}\right)^{-1} \Omega_{\infty} \subset \Omega_{\infty}$.

Proof. Let $x \in\left(f^{S_{k}(\omega)} \mid \omega^{\prime}\right)^{-1} \Omega_{\infty}$. Since $\omega^{\prime} \cap \Omega_{\infty} \neq \emptyset, x \in \Omega_{S_{k}(\omega)-1}$ holds. Since $f^{S_{k}(\omega)} x \in \Omega_{\infty}$, $x \in \Omega_{S_{k}(\omega)}$ holds. Reasoning as in the proof of Sublemma 3.4. for every $n>0$ we have $\left|f^{n+S_{k}(\omega)} x\right| \geq \delta e^{-\varepsilon n}>\delta e^{-\varepsilon\left(n+S_{k}(\omega)\right)}$, and thus $x \in \Omega_{n+S_{k}(\omega)}$. This yields $x \in \Omega_{\infty}$.

In subsequent steps we treat points sent into gaps in the previous steps. This completes the construction of $\mathcal{Q}$. (a) is a direct consequence of the construction. Since $f^{R(\omega)}=3 \Lambda^{ \pm}$ and $f^{R(\omega)} \mid \omega$ is extended to a diffeomorphism onto $3 \Lambda^{ \pm}$, the Koebe Principle [25, Chap. IV.1] yields (b).

Define an induced map $F: \Omega_{\infty} \circlearrowleft$ by $F \mid \omega=f^{R(\omega)}$ for $\omega \in \mathcal{Q}$. By Lemma 3.3 and [25, Chap. V.2 Thm. 2.2], there exists an $F$-invariant probability measure $\nu_{0}$ that is absolutely continuous with respect to the Lebesgue measure $\operatorname{Leb}_{\Omega_{\infty}}$ on $\Omega_{\infty}$, with the density $d \nu_{0} / d \operatorname{Leb}_{\Omega_{\infty}}$ uniformly bounded away from zero and infinity. The next measure estimate of the tail $\{R>$ $n\}=\left\{x \in \Omega_{\infty}: R(x)>n\right\}$ implies that $\nu_{0}$ projects down to the acip $\mu$ for $f$.

Lemma 3.6. For all large $n,|\{R>n\}| \leq e^{-\frac{\lambda}{10} n}$.

Proof. For $0 \leq k \leq n$, let

$$
\mathcal{P}_{k}^{\prime}=\left\{\omega \in \tilde{\mathcal{P}}_{k}: \omega \cap\{R>n\} \neq \emptyset\right\}
$$


Let $\omega \in \mathcal{P}_{k}^{\prime}$. By construction, all points in $\omega$ share the same sequence of regular return times up to time $k$, which we simply denote by $0=: R_{0}<R_{1}<R_{2}<\cdots \leq k$. For $i \geq 0$ let $\omega_{i}$ denote the element of $\tilde{\mathcal{P}}_{R_{i}}$ which contains $\omega$.

Sublemma 3.7. $R_{i+1}-R_{i} \geq N$ and $\left|\omega_{i+1}\right| /\left|\omega_{i}\right| \leq 3 e^{-\frac{\lambda}{3}\left(R_{i+1}-R_{i}\right)}$.

Proof. The first inequality follows from $p\left(f^{R_{i}} \omega_{i}\right) \geq N$ and that $f^{R_{i+1}} \omega_{i}$ is free. The mean value theorem gives $\left|\Lambda^{+}\right|=\left|f^{R_{i}} \omega_{i}\right|=\left|D f^{R_{i}}(x)\right|\left|\omega_{i}\right|$ for some $x \in \omega_{i}$, and $\left|f^{R_{i}} \omega_{i+1}\right|=$ $\left|D f^{R_{i}}(y)\right|\left|\omega_{i+1}\right|$ for some $y \in \omega_{i+1}$. The Koebe Principle implies $\left|D f^{R_{i}}(x)\right| \leq 3\left|D f^{R_{i}}(y)\right|$, and thus $\left|\omega_{i+1}\right| /\left|\omega_{i}\right| \leq 3\left|f^{R_{i}} \omega_{i+1}\right| /\left|\Lambda^{+}\right|$. In addition $\left|\Lambda^{+}\right|=\left|f^{R_{i+1}-R_{i}}\left(f^{R_{i}} \omega_{i+1}\right)\right| \geq e^{\frac{\lambda}{3}\left(R_{i+1}-R_{i}\right)}\left|f^{R_{i}} \omega_{i+1}\right|$, and thus the second inequality holds.

Let $1 \leq j \leq[n / N]$. For a $j$-string $\left(k_{1}, \ldots, k_{j}\right)$ of positive integers, let

$$
\mathcal{Q}\left(k_{1}, \ldots, k_{j}\right)=\left\{\omega \in \mathcal{P}_{\sum_{i=1}^{j} k_{i}}^{\prime}: R_{i}(\omega)=k_{1}+k_{2}+\cdots+k_{i} \text { for every } 1 \leq i \leq j\right\},
$$

and let $\left|\mathcal{Q}\left(k_{1}, \ldots, k_{j}\right)\right|=\sum_{\omega \in \mathcal{Q}\left(k_{1}, \ldots, k_{j}\right)}|\omega|$.

Sublemma 3.8. $\left|\mathcal{Q}\left(k_{1}, \ldots, k_{j}\right)\right| \leq e^{-\frac{\lambda}{4} \sum_{i=1}^{j} k_{i}}$.

Proof. For each $1 \leq i<j$ and $\omega_{i} \in \mathcal{Q}\left(k_{1}, \ldots, k_{i}\right)$, let

$$
\mathcal{Q}\left(\omega_{i}, k_{i+1}\right)=\left\{\omega_{i+1} \in \mathcal{Q}\left(k_{1}, \ldots, k_{i+1}\right): \omega_{i+1} \subset \omega_{i}\right\} .
$$

Using the second inequality of Sublemma 3.7 and $\# \mathcal{Q}\left(\omega_{i}, k_{i+1}\right) \leq e^{6 \varepsilon k_{i+1}}$ which follows from the proof of Lemma 2.8 we get

$$
\begin{aligned}
\left|\mathcal{Q}\left(k_{1}, \ldots, k_{i+1}\right)\right| & =\sum_{\omega_{i} \in \mathcal{Q}\left(k_{1}, \ldots, k_{i}\right)}\left|\omega_{i}\right| \sum_{\omega_{i+1} \in \mathcal{Q}\left(\omega_{i}, k_{i+1}\right)} \frac{\left|\omega_{i+1}\right|}{\left|\omega_{i}\right|} \\
& \leq \sum_{\omega_{i} \in \mathcal{Q}\left(k_{1}, \ldots, k_{i}\right)}\left|\omega_{i}\right| e^{6 \varepsilon k_{i+1}} \cdot 3 e^{-\frac{\lambda}{3} k_{i+1}} \\
& \leq e^{-\frac{\lambda}{4} k_{i+1}} \sum_{\omega_{i} \in \mathcal{Q}\left(k_{1}, \ldots, k_{i}\right)}\left|\omega_{i}\right|=e^{-\frac{\lambda}{4} k_{i+1}}\left|\mathcal{Q}\left(k_{1}, \ldots, k_{i}\right)\right|
\end{aligned}
$$

Using this inductively, combining the result with $\left|\mathcal{Q}\left(k_{1}\right)\right| \leq e^{-\frac{\lambda}{4} k_{1}}$ and then substituting $i=j-1$ we obtain the desired inequality.

Sublemma 3.9. If $\sum_{i=1}^{j} k_{i}<[n / 2]$, then $\left|\mathcal{Q}\left(k_{1}, \ldots, k_{j}\right)\right| \leq e^{-\frac{\lambda}{5} n}$.

Proof. Let $\omega \in \mathcal{Q}\left(k_{1}, \ldots, k_{j}\right)$. If $f^{n} \omega$ is bound, then let $k<n$ denote the free return with bound period $p$ with $k<n<k+p$. Since $\omega$ intersects $\Omega_{\infty}, d\left(0, f^{k} \omega\right) \geq \delta e^{-\varepsilon k}$, and thus $k+p-$ $n \leq \frac{3 \varepsilon}{\lambda} n$. Then for all $x \in \omega,\left|D f^{n}(x)\right|=\left|D f^{k+p}(x)\right| /\left|D f^{k+p-n}\left(f^{n} x\right)\right| \geq 4^{-(k+p-n)} e^{\frac{\lambda}{3}(k+p)} \geq$ $4^{-\frac{3 \varepsilon}{\lambda} n} e^{\frac{\lambda}{3} n} \geq e^{\frac{\lambda}{4} n}$, and thus $|\omega| \leq e^{-\frac{\lambda}{4} n}$. If $f^{n} \omega$ is free, then $|\omega| \leq e^{-\frac{\lambda}{4} n}$. Hence

$$
\left|\mathcal{Q}\left(k_{1}, \ldots, k_{j}\right)\right| \leq e^{-\frac{\lambda}{4} n} \# \mathcal{Q}\left(k_{1}, \ldots, k_{j}\right) .
$$

From the proof of Lemma 2.8 and the assumption $\sum_{i=1}^{j} k_{i}<[n / 2]$ we have $\# \mathcal{Q}\left(k_{1}, \ldots, k_{j}\right) \leq$ $e^{5 \varepsilon \sum_{i=1}^{j} k_{i}} \leq e^{\frac{5 \varepsilon}{2} n}$, and so the desired inequality follows. 
Returning to the proof of Lemma 3.6. let $\mathcal{Q}_{n}^{j}$ denote the collection of elements of $\mathcal{P}_{n}^{\prime}$ for which the number of regular return times up to time $n$ is equal to $j$. Let $\left|\mathcal{Q}_{n}^{j}\right|=\sum_{\omega \in \mathcal{Q}_{n}^{j}}|\omega|$. Sublemma 3.8 and Sublemma 3.9 yield

$$
\begin{aligned}
\left|\mathcal{Q}_{n}^{j}\right| & =\sum_{K=1}^{[n / 2]-1} \sum_{k_{1}+\cdots+k_{j}=K}\left|\mathcal{Q}\left(k_{1}, \ldots, k_{j}\right)\right|+\sum_{K=[n / 2]}^{n} \sum_{k_{1}+\cdots+k_{j}=K}\left|\mathcal{Q}\left(k_{1}, \ldots, k_{j}\right)\right| \\
& \leq e^{-\frac{\lambda}{5} n} \sum_{K=1}^{[n / 2]-1} \#\left\{\left(k_{1}, \ldots, k_{j}\right): \sum_{i=1}^{j} k_{i}=K\right\} \\
& +\sum_{K=[n / 2]}^{n} e^{-\frac{\lambda}{4} K} \#\left\{\left(k_{1}, \ldots, k_{j}\right): \sum_{i=1}^{j} k_{i}=K\right\} \\
& \leq e^{-\frac{\lambda}{5} n} \sum_{K=1}^{[n / 2]-1} e^{\beta K}+\sum_{K=[n / 2]}^{n} e^{-\left(\frac{\lambda}{4}-\beta\right) K},
\end{aligned}
$$

where $\beta \rightarrow 0$ as $N \rightarrow \infty$. Hence $\left|\mathcal{Q}_{n}^{j}\right| \leq e^{-\frac{\lambda}{9} n}$ and $|\{R>n\}| \leq \sum_{j=1}^{[n / N]}\left|\mathcal{Q}_{n}^{j}\right| \leq e^{-\frac{\lambda}{10} n}$.

\subsection{Reduction to lower floors of the tower. Let}

$$
\Delta=\left\{(x, l): x \in \Omega_{\infty}, \quad l=0,1, \ldots, R(x)-1\right\},
$$

which we call a tower, and define

$$
\hat{f}(x, l)=\left\{\begin{array}{l}
(x, l+1) \text { if } l+1<R(x) \\
\left(f^{R(x)} x, 0\right) \text { if } l+1=R(x) .
\end{array}\right.
$$

The point $(x, l)$ is considered to be climbing the tower in the first case and falling down from the tower in the second case. Define a projection $\pi: \Delta \rightarrow X$ by $\pi(x, l)=f^{l} x$. Let $\Delta_{l}=\{(x, l) \in \Delta: R(x)>l\}$. Note that $\Delta_{0}=\left\{(x, 0): x \in \Omega_{\infty}\right\}$. Let $\tau_{l}:\{R>l\} \rightarrow \Delta_{l}$ denote the canonical identification $\tau_{l}(x)=(x, l)$. Fix a measurable structure on $\Delta$ such that $\pi$ is measurable, and define a probability measure $\hat{\mu}$ on $\Delta$ by

$$
\hat{\mu}=\frac{1}{\nu_{0}(R)} \sum_{l=0}^{\infty}\left(\tau_{l}\right)_{*} \nu_{0} \mid\{R>l\}
$$

where $\nu_{0}(R)<\infty$ by Lemma 3.6. Observe that $\pi_{*} \hat{\mu}=\mu$.

We reduce the desired upper estimate in the proposition to an estimate on the lower floors of the tower. For each $l \geq 0$, let $\mathcal{P}_{l}=\tilde{\mathcal{P}}_{l} \mid\{R>l\}$. Using $\tau_{l}$ we transplant the partition $\mathcal{P}_{l}$ to $\Delta_{l}$ and also denote it by $\mathcal{P}_{l}$. Let $\mathcal{D}=\bigcup_{l \geq 0} \mathcal{P}_{l}$ denote the resultant partition of $\Delta$. Let $\hat{\varphi}_{j}=\varphi_{j} \circ \pi$. Let

$$
\mathcal{B}_{n}=\left\{A \in \bigvee_{i=0}^{n-1} \hat{f}^{-i} \mathcal{D}: \frac{1}{n} S_{n} \hat{\varphi}_{j}(x) \geq b_{j} \quad j=1, \ldots, d \text { for some } x \in A\right\} .
$$

If $\mathcal{B}_{n}=\emptyset$ there is nothing to prove, and hence we assume $\mathcal{B}_{n} \neq \emptyset$. We have

$$
\frac{1}{n} \log \mu\left\{\frac{1}{n} S_{n} \varphi_{j} \geq b_{j}\right\}=\frac{1}{n} \log \hat{\mu}\left\{\frac{1}{n} S_{n} \hat{\varphi}_{j} \geq b_{j}\right\} \leq \frac{1}{n} \log \hat{\mu}\left(\mathcal{B}_{n}\right),
$$


where $\hat{\mu}\left(\mathcal{B}_{n}\right)=\sum_{A \in \mathcal{B}_{n}} \hat{\mu}(A)$. Define

$$
\mathcal{B}_{n}^{\prime}=\left\{A \in \mathcal{B}_{n}: A \subset \bigcup_{0 \leq l \leq 30 n} \Delta_{l}\right\} \text { and } \mathcal{B}_{n}^{\prime \prime}=\left\{A \in \mathcal{B}_{n}: A \subset \bigcup_{l>30 n} \Delta_{l}\right\} .
$$

Let $p_{1}: \Delta \rightarrow \Lambda$ denote the projection to the first coordinate. The next lemma enables us to compare $\hat{\mu}$ and $\operatorname{Leb}_{\Omega_{\infty}}$.

Lemma 3.10. There exist $0<C_{1}<C_{2}$ such that for any $l \geq 0$ and any measurable set $A \subset \Delta_{l}$ we have $C_{1}\left|p_{1} A\right| \leq \hat{\mu}(A) \leq C_{2}\left|p_{1} A\right|$.

Proof. For any measurable $A \subset \Delta_{l}$ we have $\hat{\mu}(A)=\nu_{0}\left(p_{1} A\right) / \nu_{0}(R)$, and the density of $\nu_{0}$ is uniformly bounded away from zero and infinity. Hence the claim holds.

By Lemma 3.6 and Lemma 3.10,

$$
\hat{\mu}\left(\mathcal{B}_{n}^{\prime \prime}\right) \leq C_{2} \sum_{l>30 n}|\{R>l\}|<4^{-n}
$$

and thus for any $\nu \in \mathcal{M}_{f}$,

$$
\varlimsup_{n \rightarrow \infty} \frac{1}{n} \log \hat{\mu}\left(\mathcal{B}_{n}^{\prime \prime}\right) \leq-\log 4 \leq F(\nu) .
$$

For the proof of the proposition it suffices to show that for all large $n$ such that $\mathcal{B}_{n}^{\prime} \neq \emptyset$ there exists $\sigma \in \mathcal{M}_{f}$ satisfying (2) such that

$$
\frac{1}{n} \log \hat{\mu}\left(\mathcal{B}_{n}^{\prime}\right) \leq\left(1-\varepsilon^{1 / 5}\right) F(\sigma)+2 \varepsilon^{1 / 5} .
$$

For the rest of this paper we assume $\mathcal{B}_{n}^{\prime} \neq \emptyset$.

3.4. Approximation by points quickly falling down from the tower. For $n \geq N$ and $\omega \in \mathcal{P}_{n}$, let $\tilde{\omega}$ denote the element of $\tilde{\mathcal{P}}_{n}$ containing $\omega$, namely $\omega=\tilde{\omega} \cap\{R>n\}$. Points in $\omega$ may climb the tower for a very long period of time. The next lemma indicates that a positive definite fraction of points in $\tilde{\omega}$ quickly fall down to the ground floor $\Delta_{0}$.

Lemma 3.11. There exists $n_{0}^{\prime \prime} \geq n_{0}^{\prime}$ such that for every $n \geq n_{0}^{\prime \prime}$ and $\omega \in \mathcal{P}_{n}$ there exist $\omega^{\prime} \subset \tilde{\omega} \cap \Omega_{\infty}$ and $r=r\left(\omega^{\prime}\right) \in\left[n,\left(1+\varepsilon^{1 / 3}\right) n\right]$ such that:

(a) $\left|\omega^{\prime}\right| \geq e^{-\varepsilon^{1 / 3} n}|\tilde{\omega}|$;

(b) $f^{r}$ maps $\omega^{\prime}$ bijectively onto $\Omega_{\infty}^{ \pm}$.

Proof. Let $\hat{n}=\min \left\{i \geq n: f^{i} \tilde{\omega}\right.$ is free $\}$.

Sublemma 3.12. $\left|\left\{S_{e(\tilde{\omega})}<\hat{n}+\varepsilon^{1 / 2} n\right\} \cap \tilde{\omega} \cap \Omega_{\infty}\right| \geq(1 / 3)|\tilde{\omega}|$.

Proof. If the reverse inequality were true, then using $\left|\tilde{\omega} \backslash \Omega_{\infty}\right| \leq(1 / 2)|\tilde{\omega}|$ which follows from Lemma 3.1 we would get $\left|\left\{S_{e(\tilde{\omega})}<\hat{n}+\varepsilon^{1 / 2} n \mid \tilde{\omega}\right\}\right|<1 / 2+1 / 3$. This yields a contradiction to $\left|\left\{S_{e(\tilde{\omega})} \geq \hat{n}+\varepsilon^{1 / 2} n \mid \tilde{\omega}\right\}\right| \leq C \zeta^{n}$ obtained from Lemma 2.8 provided $n$ is sufficiently large.

By Sublemma 3.12 and \# $\left\{\omega^{\prime} \in \mathcal{F}_{e(\tilde{\omega})} \mid \tilde{\omega}: S_{e(\tilde{\omega})}\left(\omega^{\prime}\right)<\hat{n}+\varepsilon^{1 / 2} n\right\} \leq \sum_{i=0}^{\left[\varepsilon^{1 / 2} n\right]} 2^{i+1} \leq 3^{\varepsilon n}$, one can choose an integer $r \in\left[\hat{n}, \hat{n}+\varepsilon^{1 / 2} n\right] \subset\left[n,\left(1+\varepsilon^{1 / 3}\right) n\right]$ and $\omega_{0} \in \mathcal{F}_{e(\tilde{\omega})} \mid \tilde{\omega}$ intersecting $\Omega_{\infty}$ such that $S_{e(\tilde{\omega})}\left(\omega_{0}\right)=r$ and $\left|\omega_{0}\right| \geq(1 / 3) 3^{-\varepsilon^{1 / 2} n}|\tilde{\omega}|$. Define $\omega^{\prime} \subset \omega_{0}$ by $\omega^{\prime}=\left(f^{r} \mid \omega_{0}\right)^{-1} \Omega_{\infty}^{ \pm}$if $f^{r} \omega_{0}=\Lambda^{ \pm}$, respectively. Since $\omega_{0} \cap \Omega_{\infty} \neq \emptyset, \omega_{0} \subset \Omega_{r}$. This and $f^{r} \omega_{0}=\Lambda^{ \pm}$together imply 
$\omega_{0} \subset \Omega_{\infty}$. The bounded distortion in Lemma 2.7 and Lemma 3.1 yield $\left|\omega^{\prime}\right| \geq\left(C_{\varepsilon}^{-1} / 2\right)\left|\omega_{0}\right| \geq$ $e^{-\varepsilon^{1 / 3} n}|\tilde{\omega}|$. Hence (a) holds. (b) is obvious from the construction.

3.5. Construction of a horseshoe. Let $L_{1}, \ldots, L_{q}$ be a collection of pairwise disjoint closed intervals in $\left[f^{2} 0, f 0\right]$ and $m$ a positive integer. We say $\left\{L_{i}\right\}_{i=1}^{q}$ generates a horseshoe for $f^{m}$ if $f^{m}$ maps each $L_{i}(1 \leq i \leq q)$ diffeomorphically onto the same interval containing $\bigcup_{i=1}^{q} L_{i}$ in its interior.

Lemma 3.13. For all large $n$ there exist a collection $L_{1}, \ldots, L_{q}$ of closed intervals and an integer $m \in\left[\left(1-\varepsilon^{1 / 5}\right) n,\left(1+\varepsilon^{1 / 5}\right) n\right]$ such that:

(a) $\left\{L_{i}\right\}_{i=1}^{q}$ generates a horseshoe for $f^{m}$;

(b) $\sum_{i=1}^{q}\left|L_{i}\right| \geq e^{-\varepsilon^{1 / 5} n} \hat{\mu}\left(\mathcal{B}_{n}^{\prime}\right)$;

(c) for all $x \in \bigcap_{j=0}^{\infty}\left(f^{m}\right)^{-j}\left(\bigcup_{i=1}^{q} L_{i}\right),(1 / m) S_{m} \varphi_{j}(x) \geq b_{j}-\varepsilon^{1 / 2}, j=1, \ldots, d$.

Proof. Let $A \in \mathcal{B}_{n}^{\prime}$ and $l_{A} \geq 0$ be such that $A \subset \Delta_{l_{A}}$. In the first $n$-iterates under $\hat{f}$, the set $A$ continues climbing the tower, or else falls down from the tower several times. Hence, there exists an integer $k_{A} \in[0, n-1] \cup\left\{n+l_{A}\right\}$ such that $\hat{f}^{n} A \in \mathcal{D} \mid \Delta_{k_{A}}$. Thus $p_{1}\left(\hat{f}^{n} A\right)$ is an element of $\mathcal{P}_{k_{A}}$, which we denote by $\omega_{A}$. If $k_{A} \geq n_{0}^{\prime \prime}$, then take $\omega_{A}^{\prime} \subset \tilde{\omega}_{A} \in \tilde{\mathcal{P}}_{k_{A}}$ for which the conclusions of Lemma 3.11 hold. Define an interval $\tilde{A}$ containing $p_{1} A$ so that: $\tilde{A}=\tilde{\omega}_{A}$ if $k_{A}=n+l_{A} ; f^{n-k_{A}+l_{A}} \tilde{A}=\tilde{\omega}_{A}$ if $n_{0}^{\prime \prime} \leq k_{A} \leq n-1 ; f^{n-k_{A}+l_{A}} \tilde{A}=\Lambda^{ \pm}$if $k_{A}<n_{0}^{\prime \prime}$. Set

$$
\ell_{A}=\min \left\{j \geq l_{A}: f^{j} \tilde{A} \text { is free }\right\},
$$

and define

$$
t_{A}= \begin{cases}n-k_{A}+r\left(\omega_{A}^{\prime}\right)-\ell_{A}+l_{A} & \text { if } k_{A} \geq n_{0}^{\prime \prime} \\ n-k_{A}-\ell_{A}+l_{A} & \text { if } k_{A}<n_{0}^{\prime \prime} .\end{cases}
$$

Sublemma 3.14. For any $A \in \mathcal{B}_{n}^{\prime},\left(1-\varepsilon^{1 / 2}\right) n \leq t_{A} \leq\left(1+\varepsilon^{1 / 4}\right) n$.

Proof. Lemma 3.11 gives $k_{A} \leq r\left(\omega_{A}^{\prime}\right) \leq\left(1+\varepsilon^{1 / 3}\right) k_{A}$. By construction and Lemma 2.3(b), $l_{A} \leq \ell_{A} \leq(1+6 \varepsilon) l_{A}$. Hence, if $k_{A} \geq n_{0}^{\prime \prime}$ then

$$
t_{A} \leq n-k_{A}+r\left(\omega_{A}^{\prime}\right) \leq n+\varepsilon^{1 / 3} k_{A} \leq\left(1+31 \varepsilon^{1 / 3}\right) n<\left(1+\varepsilon^{1 / 4}\right) n,
$$

where the third inequality follows from $k_{A} \leq n+l_{A}$ and $l_{A} \leq 30 n$. On the other hand,

$$
t_{A} \geq n-6 \varepsilon l_{A} \geq(1-180 \varepsilon) n \geq\left(1-\varepsilon^{1 / 2}\right) n .
$$

If $k_{A}<n_{0}^{\prime \prime}$, then clearly $t_{A} \leq n$, and

$$
t_{A} \geq n-k-6 \varepsilon l_{A} \geq(1-180 \varepsilon) n-n_{0}^{\prime \prime} \geq\left(1-\varepsilon^{1 / 2}\right) n,
$$

where the last inequality holds provided $n$ is sufficiently large.

By construction, for any $A \in \mathcal{B}_{n}^{\prime}$ one can choose a set $A^{\prime} \subset \Delta_{l_{A}}$ so that: $p_{1}\left(\hat{f}^{n} A^{\prime}\right)=\omega_{A}^{\prime}$ if $k_{A}=n+l_{A} ; p_{1}\left(\hat{f}^{n-k_{A}} A^{\prime}\right)=\omega_{A}^{\prime}$ if $n_{0}^{\prime \prime} \leq k_{A} \leq n-1 ; A \subset A^{\prime}$ and $p_{1}\left(\hat{f}^{n-k_{A}} A^{\prime}\right)=\Omega_{\infty}^{ \pm}$if $k_{A}<n_{0}^{\prime \prime}$.

Sublemma 3.15. For any $A \in \mathcal{B}_{n}^{\prime}, \hat{\mu}\left(A^{\prime}\right) \geq e^{-\varepsilon^{1 / 4} n} \hat{\mu}(A)$.

Proof. Lemma 3.10 gives

$$
\frac{\hat{\mu}\left(A^{\prime}\right)}{\hat{\mu}(A)} \geq C_{1} C_{2}^{-1} \frac{\left|p_{1} A^{\prime}\right|}{\left|p_{1} A\right|} .
$$


As for the fraction of the right-hand-side, if $k_{A}=n+l_{A}$, then using $\omega_{A} \subset \tilde{\omega}_{A}$ and Lemma 3.11 we have

$$
\frac{\left|p_{1} A^{\prime}\right|}{\left|p_{1} A\right|}=\frac{\left|\omega_{A}^{\prime}\right|}{\left|\omega_{A}\right|} \geq \frac{\left|\omega_{A}^{\prime}\right|}{\left|\tilde{\omega}_{A}\right|} \geq e^{-\varepsilon^{1 / 3}\left(n+l_{A}\right)} \geq e^{-31 \varepsilon^{1 / 3} n} .
$$

If $n_{0}^{\prime \prime} \leq k_{A} \leq n-1$, then additionally using the bounded distortion we have

$$
\frac{\left|p_{1} A^{\prime}\right|}{\left|p_{1} A\right|} \geq C_{\varepsilon}^{-1} \frac{\left|p_{1}\left(\hat{f}^{n-k} A^{\prime}\right)\right|}{\left|p_{1}\left(\hat{f}^{n-k} A\right)\right|}=C_{\varepsilon}^{-1} \frac{\left|\omega_{A}^{\prime}\right|}{\left|\omega_{A}\right|} \geq C_{\varepsilon}^{-1} \frac{\left|\omega_{A}^{\prime}\right|}{\left|\tilde{\omega}_{A}\right|} \geq C_{\varepsilon}^{-1} e^{-31 \varepsilon^{1 / 3} n} .
$$

If $k_{A}<n_{0}^{\prime \prime}$, then $\frac{\left|p_{1} A^{\prime}\right|}{\left|p_{1} A\right|} \geq 1$ because $A \subset A^{\prime}$. Consequently the desired inequality holds provided $n$ is sufficiently large.

Returning to the proof of Lemma 3.13 , choose $m_{0} \in\left[\left(1-\varepsilon^{1 / 2}\right) n,\left(1+\varepsilon^{1 / 4}\right) n\right]$ such that

$$
\sum_{A \in \mathcal{B}_{n}^{\prime}: t_{A}=m_{0}} \hat{\mu}\left(A^{\prime}\right) \geq \frac{1}{2 \varepsilon^{1 / 4} n} \sum_{A \in \mathcal{B}_{n}^{\prime}} \hat{\mu}\left(A^{\prime}\right) .
$$

Set $q=\#\left\{A \in \mathcal{B}_{n}^{\prime}: t_{A}=m_{0}\right\}$ and $\left\{A_{i}\right\}_{i=1}^{q}=\left\{A \in \mathcal{B}_{n}^{\prime}: t_{A}=m_{0}\right\}$. For each $i \in[1, q]$ let $\hat{K}_{i}$ denote the smallest closed interval containing $p_{1} A_{i}^{\prime}$, and define $K_{i}=f^{\ell_{A_{i}}} \hat{K}_{i}$. Then $K_{i} \subset\left(f^{2} 0, f 0\right)$, and $f^{m_{0}} K_{i}=\Lambda^{ \pm}$because $f^{t_{A}}\left(f^{\ell_{A}} A^{\prime}\right)=\Omega_{\infty}^{ \pm}$for any $A \in \mathcal{B}_{n}^{\prime}$. By (A4) it is possible to choose $m_{1}>0$ and two closed intervals $I^{ \pm} \subset \Lambda^{ \pm}$such that $f^{m_{1}}$ maps $I^{ \pm}$ diffeomorphically onto the same interval containing $K_{1}, \ldots, K_{q}$. Define $L_{i}=\left(f^{m_{0}} \mid K_{i}\right)^{-1} I^{ \pm}$ if $f^{m_{0}} K_{i}=\Lambda^{ \pm}$, respectively. By construction, $L_{1}, \ldots, L_{q}$ are pairwise disjoint. Then $m=$ $m_{0}+m_{1} \in\left[\left(1-\varepsilon^{1 / 5}\right) n,\left(1+\varepsilon^{1 / 5}\right) n\right]$ holds for sufficiently large $n$, and $\left\{L_{i}\right\}_{i=1}^{q}$ generates a horseshoe for $f^{m}$. In addition, by Lemma 3.10, (18) and Sublemma 3.15,

$$
\sum_{A \in \mathcal{B}_{n}^{\prime}: t_{A}=m_{0}} \hat{\mu}\left(A^{\prime}\right)=\sum_{i=1}^{q} \hat{\mu}\left(A_{i}^{\prime}\right) \geq \frac{1}{2 \varepsilon^{1 / 4} n} e^{-\varepsilon^{1 / 4} n} \hat{\mu}\left(\mathcal{B}_{n}^{\prime}\right) \geq e^{-\varepsilon^{1 / 5} n} \hat{\mu}\left(\mathcal{B}_{n}^{\prime}\right) .
$$

Since both $f^{\ell_{A_{i}}} \hat{K}_{i}$ and $\hat{K}_{i}$ are free, $\left|D f^{\ell_{A_{i}}}\right| \geq \delta$ on $\hat{K}_{i}$. Hence $\left|K_{i}\right|=\left|f^{\ell_{A_{i}}} \hat{K}_{i}\right| \geq \delta\left|\hat{K}_{i}\right| \geq$ $\delta\left|p_{1} A_{i}^{\prime}\right| \geq C_{2}^{-1} \delta \cdot \hat{\mu}\left(A_{i}^{\prime}\right)$. Using this and the bounded distortion we get $\left|L_{i}\right| \geq C_{\varepsilon}^{-1} \frac{\left|I^{+}\right|}{\left|\Lambda^{+}\right|}\left|K_{i}\right| \geq$ $C_{\varepsilon}^{-1} \frac{\left|I^{+}\right|}{\left|\Lambda^{+}\right|} C_{2}^{-1} \delta \cdot \hat{\mu}\left(A_{i}^{\prime}\right)$. Plugging this estimate into the left-hand-side of (19) yields Lemma $3.13(\mathrm{~b})$.

For the proof of Lemma 3.13 (c) it suffices to show $S_{m} \hat{\varphi}_{j}(x) \geq\left(b_{j}-\varepsilon^{1 / 2}\right) m$ for all $x \in$ $\bigcup_{i=1}^{q}(\hat{f})^{l_{i}-l_{A_{i}}} A_{i}$. Pick $x_{i} \in A_{i} \in \mathcal{B}_{n}$ such that $S_{n} \hat{\varphi}\left(x_{i}\right) \geq b_{j} n$ holds for $j=1, \ldots, d$. We have

$$
\left|S_{m} \hat{\varphi}_{j}\left(\hat{f}^{l_{i}-l_{A_{i}}} x_{i}\right)-S_{n} \hat{\varphi}_{j}\left(x_{i}\right)\right| \leq\left(2\left(l_{i}-l_{A_{i}}\right)+|m-n|\right)\left\|\varphi_{j}\right\|,
$$

where $\left\|\varphi_{j}\right\|=\sup \left|\varphi_{j}\right|$. Since $\left|l_{i}-l_{A_{i}}\right| \leq \frac{3 \varepsilon}{\lambda} l_{A_{i}} \leq \varepsilon^{2 / 3} n$ and $|m-n| \leq \varepsilon^{2 / 3} n$ we have

$$
S_{m} \hat{\varphi}_{j}\left(\hat{f}^{l_{i}-l_{A_{i}}} x_{i}\right) \geq S_{n} \hat{\varphi}_{j}\left(x_{i}\right)-\left(2\left(l_{i}-l_{A_{i}}\right)+|m-n|\right)\left\|\varphi_{j}\right\| \geq b_{j} n-2 \varepsilon^{2 / 3} n .
$$

Hence, for each $i=1, \ldots, q$ and $j=1, \ldots, d$,

$$
S_{m} \hat{\varphi}_{j}\left(\hat{f}^{l_{i}-l_{A_{i}}} x_{i}\right) \geq\left(b_{j}-\varepsilon^{1 / 2} / 2\right) m .
$$

Sublemma 3.16. For any $n \geq 1$ and $\omega \in \tilde{\mathcal{P}}_{n-1}, \sum_{i=0}^{n-1}\left|f^{i} \omega\right| \leq 10 \delta^{-1}$. 
Proof. Let $n_{1}<\cdots<n_{s}<n$ denote all the free returns in the first $n-1$-iterates of $\omega$, with $p_{1}, \ldots, p_{s}$ the corresponding bound periods. Let $1 \leq i \leq s$. For each $j \in\left[n_{i}+1, n_{i}+p_{i}-1\right]$, choose $\theta_{j} \in f^{n_{i}} \omega$ such that $\left|f^{j} \omega\right|=\left|f^{n_{i}} \omega\right| \cdot\left|D f^{j-n_{i}}\left(\theta_{j}\right)\right|$. Then $\left|f^{j-n_{i}} \theta_{j}-f^{j-n_{i}} 0\right| \leq e^{-\varepsilon\left(p_{i}-1\right)}$, and by the bounded distortion during the bound period,

$$
\left|D f^{j-n_{i}-1}\left(f \theta_{j}\right)\right| \leq 2 \cdot \frac{\left|f^{j-n_{i}} \theta_{j}-f^{j-n_{i}} 0\right|}{\left|f \theta_{j}-f 0\right|} \leq \delta_{p_{i}}^{-2} e^{-\varepsilon\left(p_{i}-1\right)} \leq 3 \delta_{p_{i}-1}^{-2} e^{-\varepsilon\left(p_{i}-1\right)} e^{\alpha \sqrt{p_{i}}} .
$$

For the last inequality we have used (9). We also have $\left|D f\left(\theta_{j}\right)\right| \leq 4 \delta_{p_{i}-1}$. Plugging these two derivative estimates into the equality and summing the result over all $j$ gives

$$
\sum_{j=n_{i}+1}^{n_{i}+p_{i}-1}\left|f^{j} \omega\right| \leq\left|f^{n_{i}} \omega\right| \delta_{p_{i}-1}^{-1} e^{-\frac{\varepsilon}{2}\left(p_{i}-1\right)}
$$

Summing this over all $i$ gives

$$
\sum_{i=1}^{s} \sum_{j=n_{i}+1}^{n_{i}+p_{i}-1}\left|f^{j} \omega\right| \leq \sum_{i=1}^{s}\left|f^{n_{i}} \omega\right| \delta_{p_{i}-1}^{-1} e^{-\frac{\varepsilon}{2}\left(p_{i}-1\right)} \leq \sum_{p \geq N} \delta_{p-1}^{-1} e^{-\frac{\varepsilon}{2}(p-1)} \sum_{i: p_{i}=p}\left|f^{n_{i}} \omega\right| .
$$

Let $n_{i_{j}}, j=1, \ldots, t$ denote the subsequence of returns with the same bound period equal to p. By Lemma 2.3 and Lemma 2.5, for all $\theta \in f^{n_{i_{j}} \omega}$ we have $\left|D f^{n_{i_{t}}-n_{i_{j}}}(\theta)\right| \geq e^{\frac{\lambda p}{3}(t-j)}$, and thus $\left|f^{n_{i_{j}}} \omega\right| \leq e^{-\frac{\lambda p}{3}(t-j)}\left|f^{n_{i_{t}}} \omega\right|$. We also have $\mid f^{n_{i_{t}} \omega \mid} \leq 2 \delta_{p-1}$, and therefore

$$
\sum_{i: p_{i}=p}\left|f^{n_{i}} \omega\right|=\sum_{j=1}^{t}\left|f^{n_{i_{j}}} \omega\right| \leq \sum_{j=1}^{t} e^{-\frac{\lambda p}{3}(t-j)}\left|f^{n_{i_{t}}} \omega\right| \leq 2\left|f^{n_{i_{t}}} \omega\right| \leq 4 \delta_{p-1} .
$$

Substituting this estimate into the previous inequality gives

$$
\sum_{j \in \cup_{i=1}^{s}\left(n_{i}, n_{i}+p_{i}\right)}\left|f^{j} \omega\right| \leq \sum_{p \geq N} e^{-\frac{\varepsilon}{2}(p-1)} .
$$

We use part of the estimates in the proof of Lemma 2.7 to get

$$
\sum_{j \in[0, n) \backslash \cup_{i=1}^{s}\left(n_{i}, n_{i}+p_{i}\right)}\left|f^{j} \omega\right| \leq 5 \delta^{-1}\left|f^{n} \omega\right| \leq 10 \delta^{-1} .
$$

These two inequalities yield the desired one.

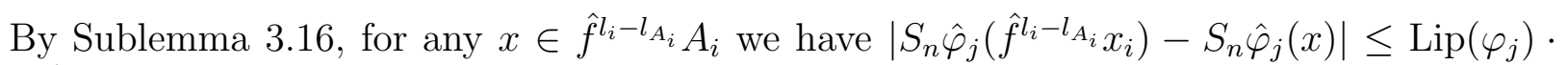
$10 \delta^{-1}$, where $\operatorname{Lip}\left(\varphi_{j}\right)$ denotes the Lipschitz constant of $\varphi_{j}$. Hence we have

$$
\left|S_{m} \hat{\varphi}_{j}\left(\hat{f}^{l_{i}-l_{A_{i}}} x_{i}\right)-S_{m} \hat{\varphi}_{j}(x)\right| \leq \operatorname{Lip}\left(\varphi_{j}\right) \cdot 10 \delta^{-1}+2\left\|\varphi_{j}\right\|(m-n) \leq\left(\varepsilon^{1 / 2} / 2\right) m .
$$

From (20), (21) we obtain $S_{m} \hat{\varphi}_{j}(x) \geq\left(b_{j}-\varepsilon^{1 / 2}\right) m$.

3.6. Construction of a measure on the horseshoe. We construct a measure for which (2) (17) hold under the assumption that $\mathcal{B}_{n}^{\prime} \neq \emptyset$. Let $L_{1}, \ldots, L_{q}$ be a collection of pairwise disjoint closed intervals and $m$ a positive integer for which the conclusions of Lemma 3.13 hold. Set $H=\bigcap_{j=0}^{\infty}\left(f^{m}\right)^{-j}\left(\bigcup_{i=1}^{q} L_{i}\right)$ and define $g=f^{m} \mid H$. The Koebe Principle implies that there exist constants $c>0$ and $\kappa>1$ such that for any $x \in H$ and every $n \geq 0,\left|D g^{n}(x)\right| \geq c \kappa^{n}$. This implies that $g: H \circlearrowleft$ is Hölder conjugate to the one-sided full shift on $q$-symbols. Define a continuous function $\Phi: H \rightarrow \mathbb{R}$ by $\Phi(x)=\log |D g(x)|$. Pick an equilibrium state of $g$ for 
the potential $-\Phi$ and denote it by $\nu_{\Phi}$. Namely, $\nu_{\Phi}$ is a $g$-invariant probability measure and satisfies

$$
h_{g}\left(\nu_{\Phi}\right)-\nu_{\Phi}(\Phi)=\sup \left\{h_{g}(\nu)-\nu(\Phi): \nu \text { is } g \text {-invariant }\right\} .
$$

Here, $h_{g}(\nu)$ denotes the entropy of $(g, \nu)$. Let $\sigma=(1 / m) \sum_{i=0}^{m-1}\left(f^{i}\right)_{*} \nu_{\Phi}$, which is $f$-invariant and ergodic. From Lemma 3.13(c) it follows that $S_{m} \varphi_{j} \geq\left(b_{j}-\varepsilon^{1 / 2}\right) m \nu_{\Phi^{-}}$a.e. Hence $\sigma\left(\varphi_{j}\right)=$ $(1 / m) \nu_{\Phi}\left(S_{m} \varphi_{j}\right) \geq b_{j}-\varepsilon^{1 / 2}$, and (2) holds.

For $k>0$ and a $(k+1)$-string $\left(a_{0}, \ldots, a_{k}\right)$ of integers in $[1, q]$, let

$$
L_{a_{0} \cdots a_{k}}=L_{a_{0}} \cap g^{-1} L_{a_{1}} \cap \cdots \cap g^{-k} L_{a_{k}} .
$$

By the Koebe Principle, there exists $\tau \in(0,1)$ such that $\left|L_{a_{0} \cdots a_{k-1} a_{k}}\right| /\left|L_{a_{0} \cdots a_{k-1}}\right| \geq \tau\left|L_{a_{k}}\right|$. Hence

$$
\begin{aligned}
\sum_{\left(a_{0}, \ldots, a_{k}\right)}\left|L_{a_{0} \cdots a_{k}}\right| & =\sum_{\left(a_{0}, \ldots, a_{k-1}\right)}\left|L_{a_{0} \cdots a_{k-1}}\right| \sum_{a_{k}} \frac{\left|L_{a_{0} \cdots a_{k-1} a_{k}}\right|}{\left|L_{a_{0} \cdots a_{k-1}}\right|} \\
& \geq \tau \sum_{j=1}^{q}\left|L_{j}\right| \sum_{\left(a_{0}, \ldots, a_{k-1}\right)}\left|L_{a_{0} \cdots a_{k-1}}\right| \geq\left(\tau \sum_{j=1}^{q}\left|L_{j}\right|\right)^{k+1}
\end{aligned}
$$

This yields

$$
\varliminf_{k \rightarrow \infty} \frac{1}{k} \log \sum_{\left(a_{0}, \ldots, a_{k}\right)}\left|L_{a_{0} \cdots a_{k}}\right| \geq \log \sum_{j=1}^{q}\left|L_{j}\right|+\log \tau .
$$

Let $\nu_{a_{0} \cdots a_{k}}$ denote the atomic probability measure equally distributed on the periodic orbit of $g$ of period $k+1$ in $L_{a_{0} \cdots a_{k}}$. Define a $g$-invariant probability measure $\nu_{k}$ by

$$
\nu_{k}=\rho_{k} \sum_{\left(a_{0}, \ldots, a_{k}\right)}\left|L_{a_{0} \cdots a_{k}}\right| \cdot \nu_{a_{0} \cdots a_{k}}
$$

where $\rho_{k}$ is the normalizing constant. Pick an accumulation point of the sequence $\left\{\nu_{k}\right\}_{k}$ and denote it by $\nu_{\infty}$. Taking a subsequence if necessary we may assume this convergence takes place for the entire sequence. By the relation $\nu_{k}\left(L_{a_{0} \cdots a_{k}}\right)=\rho_{k}\left|L_{a_{0} \cdots a_{k}}\right|$ and $\left|L_{a_{0} \cdots a_{k}}\right| \leq$ $\tau^{-1} e^{-(k+1) \nu_{a_{0} \cdots a_{k}}(\Phi)}$ we have

$$
\begin{aligned}
\log \sum_{\left(a_{0}, \ldots, a_{k}\right)}\left|L_{a_{0} \cdots a_{k}}\right| & =\sum_{\left(a_{0}, \ldots, a_{k}\right)} \nu_{k}\left(L_{a_{0} \cdots a_{k}}\right)\left(-\log \nu_{k}\left(L_{a_{0} \cdots a_{k}}\right)+\log \left|L_{a_{0} \cdots a_{k}}\right|\right) \\
& \leq-\sum_{\left(a_{0}, \ldots, a_{k}\right)} \nu_{k}\left(L_{a_{0} \cdots a_{k}}\right) \log \nu_{k}\left(L_{a_{0} \cdots a_{k}}\right)-(k+1) \nu_{k}(\Phi)-\log \tau .
\end{aligned}
$$

Then the usual proof of the variational principle [35, Theorem 9.10] shows

$$
h_{g}\left(\nu_{\infty}\right)-\nu_{\infty}(\Phi) \geq \varlimsup_{k \rightarrow \infty} \frac{1}{k} \log \sum_{\left(a_{0}, \ldots, a_{k}\right)}\left|L_{a_{0} \cdots a_{k}}\right| .
$$

Combining (22) (23) and then using Lemma 3.13(b), for all large $n$ we have

$$
h_{g}\left(\nu_{\infty}\right)-\nu_{\infty}(\Phi) \geq \log \sum_{i=1}^{q}\left|L_{i}\right|+\log \tau \geq-2 \varepsilon^{1 / 5} n+\log \hat{\mu}\left(\mathcal{B}_{n}^{\prime}\right) .
$$


Since $F(\sigma) \leq 0$ and $m \geq\left(1-\varepsilon^{1 / 5}\right) n$ we have

$$
F(\sigma) n \geq \frac{F(\sigma) m}{1-\varepsilon^{1 / 5}}=\frac{h_{g}\left(\nu_{\Phi}\right)-\nu_{\Phi}(\Phi)}{1-\varepsilon^{1 / 5}} \geq \frac{h_{g}\left(\nu_{\infty}\right)-\nu_{\infty}(\Phi)}{1-\varepsilon^{1 / 5}} \geq \frac{-2 \varepsilon^{1 / 5} n+\log \hat{\mu}\left(\mathcal{B}_{n}^{\prime}\right)}{1-\varepsilon^{1 / 5}} .
$$

Rearranging this yields (17) and hence (11).

Acknowledgments. We thank Michihiro Hirayama, Toshio Mikami, Feliks Przytycki, Juan Rivera-Letelier, Yoichiro Takahashi, Masato Tsujii and Paulo Varandas for fruitful discussions. The first-named author is partially supported by the Kyoto University Global COE Program. The second-named author is supported by the Aihara Project, the FIRST Program from the JSPS, initiated by the CSTP.

\section{REFERENCES}

[1] Araújo, V. and Pacifico, M. J.: Large deviations for non-uniformly expanding maps. J. Stat. Phys. 125, 415-457 (2006)

[2] Avila, A. and Moreira, C. G.: Statistical properties of unimodal maps: physical measures, periodic orbits and pathological laminations. Publ. Math. Inst. Hautes Étud. Sci. 101, 1-67 (2005)

[3] Baladi, V. and Viana, M.: Strong stochastic stability and rate of mixing for unimodal maps. Ann. Sci. Éc. Norm. Sup. 29, 483-517 (1996)

[4] Baxter, J. R., Jain, N. C. and Varadhan, S. R. S.: Some familiar examples for which the large deviation principle does not hold. Commun. Pure Appl. Math. 44, 911-923 (1991)

[5] Benedicks, M. and Carleson, L.: On iterations of $1-a x^{2}$ on $(-1,1)$. Ann. Math. 122, 1-25 (1985)

[6] Benedicks, M. and Carleson, L.: The dynamics of the Hénon map. Ann. Math. 133, 73-169 (1991)

[7] Benedicks, M. and Young, L.-S.: Absolutely continuous invariant measures and random perturbations for certain one-dimensional maps. Ergodic Theory and Dynamical Systems 12, 13-37 (1992)

[8] Benedicks, M. and Young, L.-S.: Markov extensions and decay of correlations for certain Hénon maps. Astérisque 261, 13-56 (2000)

[9] Bruin, H. and Keller, G.: Equilibrium states for S-unimodal maps. Ergodic Theory and Dynamical Systems 18, 765-789 (1998)

[10] Bruin, H., Luzzatto. S. and van Strien, S.: Decay of correlations in one-dimensional dynamics. Ann. Sci. Éc. Norm. Sup. 36, 621-646 (2003)

[11] Bruin, H. and Todd, M.: Equilibrium states for interval maps: the potential $-t \log D f$. Ann. Sci. Éc. Norm. Sup. 42, 559-600 (2009)

[12] Bryc, W.: On large deviations for uniformly strong mixing sequences. Stochastic Processes and their Applications 41, 191-202 (1992)

[13] Bryc, W. and Dembo, A.: Large deviations and strong mixing. Ann. Inst. Henri Poincaré 32, 549-569 (1996)

[14] Chung, Y. M.: Large deviations on Markov towers. Nonlinearity 24, 1229-1252 (2011)

[15] Comman, H. and Rivera-Letelier, J.: Large deviation principles for non-uniformly hyperbolic rational maps. Ergodic Theory and Dynamical Systems 31, 321-349 (2011)

[16] Dembo, A. and Zeitouni, O.: Large deviations techniques and Applications. 2nd ed., Applications of Mathematics 38, Springer-Verlag, New York, 1998.

[17] Donsker, M. D. and Varadhan, S. R. S.: Asymptotic evaluation of certain Markov process expectations for large time. I. II. Commun. Pure Appl. Math. 28, 1-47, 279-301 (1975)

[18] Donsker, M. D. and Varadhan, S. R. S.: Asymptotic evaluation of certain Markov process expectations for large time. III. Commun. Pure Appl. Math. 29, 389-461 (1976)

[19] Iommi, G. and Todd, M.: Dimension theory for multimodal maps. Ann. Henri Poincaré 12, 591-620 (2011)

[20] Jakobson, M.: Absolutely continuous invariant measures for one-parameter families of onedimensional maps. Commun. Math. Phys. 81, 39-88 (1981) 
[21] Keller, G. and Nowicki, T.: Spectral theory, zeta functions and the distribution of periodic points for Collet-Eckmann maps. Commun. Math. Phys. 149, 31-69 (1992)

[22] Kifer, Y.: Large deviations in dynamical systems and stochastic processes. Trans. Amer. Math. Soc. 321, 505-524 (1990)

[23] Ledrappier, F.: Some properties of absolutely continuous invariant measures of an interval. Ergodic Theory and Dynamical Systems 1, 77-93 (1981)

[24] Melbourne, I. and Nicol, M.: Large deviations for nonuniformly hyperbolic systems. Trans. Amer. Math. Soc. 360, 6661-6676 (2008)

[25] de Melo, W. and van Strien, S.: One-dimensional dynamics. Springer-Verlag, Berlin, Heidelberg, New York, 1993.

[26] Nowicki, T.: Some dynamical properties of $S$-unimodal maps. Fundamenta Math. 142, 45-57 (1993)

[27] Nowicki, T. and Sands, D.: Non-uniform hyperbolicity and universal bounds for S-unimodal maps. Invent. Math. 132, 633-680 (1998)

[28] Orey, S. and Pelikan, S.: Deviations of trajectory averages and the defect in Pesin's formula for Anosov diffeomorphisms. Trans. Amer. Math. Soc. 315, 741-753 (1989)

[29] Przytycki, F. and Rivera-Letelier, J.: Nice inducing schemes and the thermodynamics of rational maps. Commun. Math. Phys. 301, 661-707 (2011)

[30] Rey-Bellet, L. and Young, L.-S.: Large deviations in non-uniformly hyperbolic dynamical systems. Ergodic Theory and Dynamical Systems 28, 587-612 (2008)

[31] Ruelle, D.: An inequality for the entropy of differentiable maps. Bol. Soc. Brasil. Math. 9, 83-87 (1978)

[32] Takahashi, Y.: Entropy functional (free energy) for dynamical systems and their random perturbations. Stochastic analysis (Katata/Kyoto, 1982), North-Holland Math. Library 32, North-Holland, Amsterdam 437-467 (1984)

[33] Takahashi, Y.: Two aspects of large deviation theory for large time. Probabilistic methods in mathematical physics (Katata/Kyoto, 1985), Academic Press, Boston, MA, 363-384 (1987)

[34] Varandas, P.: Non-uniform specification and large deviations for weak Gibbs measures. J. Stat. Phys. 146, 330-358 (2012)

[35] Walters, P.: An introduction to ergodic theory. Graduate Texts in Mathematics 79, Springer-Verlag, New York, 1982.

[36] Young, L.-S.: Some large deviation results for dynamical systems. Trans. Amer. Math. Soc. 318, $525-543$ (1990)

[37] Young, L.-S.: Decay of correlations for certain quadratic maps. Commun. Math. Phys. 146, 123-138 (1992)

[38] Young, L.-S.: Statistical properties of dynamical systems with some hyperbolicity. Ann. Math. 147, $585-650(1998)$

[39] Young, L.-S.: Recurrence times and rates of mixing. Israel J. Math. 110, 153-188 (1999)

Department of Applied Mathematics, Hiroshima University, Higashi-Hiroshima 739-8527, JAPAN

E-mail address: chung@amath.hiroshima-u.ac.jp

First, Aihara Innovative Mathematical Modelling Project, Japan Science and Technology Agency, Institute of Industrial Science, University of Tokyo, Tokyo 153-8505, JAPAN, Current address: Department of Electronic Science and Engineering, Graduate School of EnGineERING, Kyoto University, Kyoto 606-8501, JAPAN

E-mail address: takahasi.hiroki.7r@kyoto-u.ac.jp 